\title{
Wachstum, Gewicht und Konstitution der Kinder und der herangewachsenen Jugend während des Krieges.
}

\author{
Von
}

Professor Dr. Eugen Schlesinger (Frankfurt a. M.).

(Eingegangen am 2. Mai 1919.)

Nach wie vor richten sich bei Betrachtung irgend eines Problems im Kindesalter unsere Gedanken unwillkürlich auf den Zusammenhang zwischen dem Kriege und den Kinderkrankheiten, auf den Einfluß der durch den Krieg verursachten Notlage auf unsere Jugend. Alle Bestrebungen zum Wiederaufbau der deutschen Volkskraft, unsere ganze Bevölkerungspolitik muß praktisch beim Kinde einsetzen; Voraussetzung für einen Erfolg dieser Bestrebungen ist aber die genaue Kenntnis dẹ während des Krieges und durch den Krieg hervorgerufenen Störungen und Schädigungen.

Ich habe über meine nach dieser Richtung im zweiten und dritten Kriegsjahr angestellten Untersuchungen bereits an verschiedenen Stellen berichtet: über die Schulkinder in der Münch. med. Wochenschrift 1917, S. 76 und 1505, über die herangewachsene Jugend aus der Fortbildungsschule im Archiv f. Kinderheilk. õ6, S. 161, über die Säuglinge und das Kleinkind in der Zeitschrift $\mathrm{f}$. Säuglings- und Kleinkinderschutz 1918, S.209. In dem Folgenden möchte ich meine Beobachtungen im letzten, vierten $\mathrm{K}$ riegsjahr insbesondere an den $\mathrm{K}$ naben a us den Gymnasien and Realschulen mitteilen und daran eine $Z u$ sammenfassung und die Epikrise anschließen, letztere bereits jetzt, da an meinem Beobachtungsort, StraBburg, mit dem Waffenstillstand die Kriegsnotlage zum mindesten für die Jugend bald zum $\mathrm{Ab}$ schluß kam, und ich selbst infolge meiner Ausweisung auch nicht mehr in der Lage war, an demselben Material auch noch die Reparation der festgestellten Störungen und Schädigungen zu beobachten.

Unter den einzelnen Faktoren der Kriegsnot stehen für das Kindesalter der Bedeutung nach die Ernährungsschwierigkeiten, die nach Menge und Zusammensetzung unzureichende Kriegskost weit im Vordergrund; bei der Wichtigkeit des Eiweißes für den Aufbau 
des Organismus, für das Wachstum, bei der Bedeutung des Fettes in der Nahrung für die Erhaltung der Immunität, für die Widerstandskraft gegenüber Infektionen, bei der Schädigung der Konstitution und der leichten Ủberlastung des Darmes durch die einseitige, zellulosereiche Kohlehydratkost. Von einem günstigen Einfluß der Kriegskost in der Diätetik des Kindes, insbesondere der Wohlhabenden, durch Erschwerung der Utberfütterung und Vermeidung der dadurch hervorgerufenen Äußerungen der exsudativen Diathese, konnte höchstens vorübergehend, 1916, die Rede sein. Wie sich die Unterernährung im Kindesalter in mancher Hinsicht anders äuBert als beim Erwachsenen, so z. B. in Wachstumsstörungen, so war auch das zeitliche Auftreten dieser Nährschäden deutlich verschieden; sie machten sich beim Kinde im allgemeinen erst in einer späteren Zeitperiode bemerkbar, teils schon aus äußeren Gründen, bei der in allen Volksschichten zutage getretenen Opferwilligkeit der Mütter für ihre Kinder, der reichlicheren Zuwendung einer Zusatzkost an diese, teils infolge der größeren Anpassungsfähigkeit des kindlichen Organismus, seiner leichteren Einstellung auf einen niederen Kostwert.

Gegenüber der Unterernährung tritt die mangelnde Körperpflege, der Rückgang der gesamten H ygiene bei der starken außerhäuslichen Inanspruchnahme der Mütter, die durch mancherlei Umstände bedingte größere Gefährdung durch Infektionen, die Unvollkommenheit der Erziehung infolge des Fehlens der väterlichen Autorität, des häufigen Ausfalles der Schulaufsicht, an Bedeutung zurück. Die Verkürzung des Schulbesuches mag gerade auf die körperliche Entwicklung der Kinder wohl auch günstig gewirkt haben; um so größer war aber der Ausfall an Wissen und Erziehung. Aber Sorge und Kummer und die Arbeitsüberlastung, die beim Erwachsenen durch ihre zehrende Wirkung eine so große Rolle spielen, kommen beim Kind kaum in Betracht. -

Bei der Schilderung der Wachstumshemmungen, der Änderungen des Körpergewichts, der Verschiebungen in der Konstitution des Kindes werde ich mich im wesentlichen an die rechnerisch zusammengefaßten Ergebnisse meiner uber all die Jahrefortgesetzten Reihenuntersuchungen in den Krippen, Kindergärten und insbesondere in den Schulen sämtlicher Gattungen halten und nur gelegentlich die in der offenen Kinderfürsorge, in einer Kinderpoliklinik und in der Praxis gesammelten Einzelbeobachtungen heranziehen. Aus äußeren Gründen beschränke ich mich bei der Darstellung 
auf die $\mathrm{K}$ naben und die männliche Jugend. Gerade für diese ist mein Beobachtungsmaterial ausgezeichnet durch seinen Umfang, etwa 5000 Knaben, vor allem aber auch durch seine Vollstä ndigkeit, indem es nicht nur Kinder jeder Altersstufe vom Säugling bis zum herangewachsenen 18 jährigen Jüngling, sondern auch, zum mindesten vom Schulalter ab, Kinder a us jeder Bevölkerungsschicht umfaßt, neben 330 Volksschülern und 280 Knaben aus einer gehobenen Volksschule, einer Mittelschule, und 90 Kindern aus der Hilfsschule, 700 Fortbildungsschüler, Lehrlinge im Alter von 14-17 Jahren aus der breiten Volksschicht, weiterhin auch 3000 Gymnasiasten und Realschüler aus dem oberen Mittelstand und aus den gut situierten Familien.

Als weitere Vorzüge meines Materials darf ich Nachdruck darauf legen, daß alle Untersuchungen einheitlich, von ein und de msel ben Beobachter, nach denselben Grundsätzen und Gesichtspunkten angestellt und nicht erst während des Krieges begonnen wurden, sondern schon jahrelang vorher an ebendenselben Schulen vorgenommen worden waren. So lag ein wertvolles Vergleichsmaterial vor für eine zusammenfassende Sammelstatistik, die hier, vollends bei einer möglichst weitgehenden Homogenisierung der einzelnen Gruppen, bei einer Zusammenstellung der Gruppen aus möglichst gleichartigen Individuen, vielfach wertvollere Ergebnisse lieferte als die an sich zuverlässigere Individualstatistik, die fortlaufende Beobachtung ein und desselben Kindes, welche Forschungsmethode natürlich auch ausgiebig herangezogen wurde.

Vergleiche mit den in irgend einem Lehrbuch oder Handbuch der Kinderheilkunde angegebenen Mittelzahlen (Camerer, v. Pirquet) haben gerade für den vorliegenden Zweck etwas sehr Unsicheres und führen kaum zu brauchbaren Ergebnissen. Mit der Verwertung von „Eindrücken" kann man, besonders wenn es sich um an sich nur geringe Änderungen handeln kann, wie bei der Hemmung des Wachstums, nicht vorsichtig genug sein.

Auf die Herkunft des Beobachtungsmaterials ist bei den vorliegenden Untersuchungen sehr Bedacht zu nehmen; es unterliegt wohl keinem Zweifel, daß gerade in körperlicher Hinsicht nicht die untere breite Volksmasse, sondern der Mittelstand unter dem Krieg am schwersten gelitten hat. Das gilt für die Erwachsenen wie für die Kinder. (Man vergleiche nur einmal das zweite Frühstück der Kinder aus einer Volksschule und einer Gymnasialklasse.) Auch dürfen die an einem Ort gewonnenen Untersuchungsergebnisse nicht ohne weiteres verallgemeinert 
werden; doch ist zum mindesten hinsichtlich der Großstädte eine gewisse Ubereinstimmung nicht zu verkennen. So hatte sich in Straßburg, wie wohl in der Mehrzahl der deutschen Großstädte, bereits im Laufe des Jahres 1916 die Physiognomie der Erwachsenen, besonders die der älteren Leute, wesentlich verändert, während damals und auch noch manche Monate später den Kindern noch so gut wie nichts anzusehen war. Dann war für Straßburg wie anderwärts, trotz seines agrarischen Hinterlandes, infolge der ständig starken Belegung desselben mit Truppen, das erste Halbjahr 1917 mit dem Kartoffelmangel, dem Rübenersatz und der geringen Brotration eine kritische Zeit, in der auch die Kinder sichtlich nachließen, am auffallendsten die auf ihre Ration angewiesenen Kinder in Anstalten. Aber die Säuglinge und Neugeborenen wurden auch in jener Zeit noch kaum ungünstig beeinflußt. Das Erntejahr 1917/18 brachte eine gewisse Besserung in der Nahrungsmittelversorgung der großstädtischen Bevölkerung, namentlich auch durch schärfere Erfassung der ländlichen Erzeugnisse auf dem Lande; doch konnte dadurch der bisherige Rückgang höchstens aufgehalten, keineswegs auch nur teilweise wieder gutgemacht werden. Das Jahr 1918 brachte weitere Fleischverkürzungen, eine weitere, sehr bemerkenswerte Herabsetzung der Milchration auch für die Kinder, die wohl im allgemeinen nur deshalb nicht so drückend empfunden wurde als die Verkürzungen im vorangegangenen $\mathbf{J a h r}$, weil die Kinder sich mittlerweile an den ,Mindestbedarf" angepaßt hatten. Im Sommer und Herbst 1918 wurden die gesundheitlichen Verhältnisse auch im Kindesalter vor allem von der Grippe beherrscht. Aus der sich im Innern Deutschlands anschließenden unseligen Periode der verschärften Hungerblockade mit dem Hinaufschnellen der Sterblichkeitsziffern aller Altersklassen bis zu den jüngsten Kindern fehlen mir aus den oben angeführten Gründen eigene Beobachtungen.

Die Hemmung des Längenwachstums.

Bei den Untersuchungen über etwaige durch die danernde Unterernährung zustande gekommene Wachstumsstörungen kommt zunächst und in erster Reihe nur das Längenwachstum in Betracht; denn das Massenwachstum des Körpers ist, soweit es in den Gewichtsbestimmungen zum Ausdruck kommt, mehr oder weniger verschleiert und die Feststellung seiner Größe ist unsicher gemacht durch die Änderungen im Fettansatz und im Wassergehalt des Körpers, die naturgemäß gerade bei den vorliegenden Untersuchungen ganz besonders zu berück- 
sichtigen sind. Immerhin werden die Wägungen in Verbindung mit den Längenmessungen auch hier wertvolle Aufsehlüsse über das Massenwachstum geben können. Davon im nächsten Kapitel.

Gerade für die Beurteilung des Längenwachstums kann die Sa m melstatistik bei homogener Gruppenbildung zuverlässige Ergebnisse lieferm; haben mir doch frühere Untersuchungen (Zeitschr. f. Kinderheilkunde 16, S. 265. 1917) gezeigt, daß die Durchschnittswerte der Körperlänge einer solchen homogenen Gruppe, z. B. gleichaltriger Schüler aus demselben sozialen Milieu, aus ein und derselben Schule, in den einzelnen Jahren unter normalen Verhältnissen recht konstant sind, weit konstanter als die Gewichtszahlen, und daß andererseits die Unterschiede in der Längenentwicklung bei gleichaltrigen Schülern eines auch nur wenig verschiedenen sozialen Milieus deutlicher und vor allem auch regelmäßiger sind als diejenigen der Massenzunahme.

Im allgemeinen genügen zu den Vergleichen die Durchschnittswerte; nur in besonderen Fällen, bei auffallenden Unterschieden, habe ich auch den Aufbau dieser Durchschnittszahlen, die Streuung, den prozentualen Anteil z. B. der ungewöhnlich großen oder der auffallend kleinen Kinder errechnet.

An sich ist das Längenwachstum des Kindes in seiner Art und Schnelligkeit in weitgehendem Maße von dem Umfang und der Art der Ernährung unabhängig, vielmehr vor allem bedingt durch innere, vererbte Veranlagung. Bekanntlich wird der Säugling, das Kind in der lebhaftesten Wachstumsperiode, bei schweren Ernährungsstörungen auch durch intensive Nahrungsbeschränkung auffallend wenig, langsam und spät in seinem Längenwachstum beeinflußt. Erst bei schweren infektiösen Ernährungsstörungen der Säuglinge, welche die ganze Konstitution des Kindes änderten, beobachtete Stolte und andere eine vorübergehende Hemmung, und A ron konnte zeigen, daß ernährungsgestörte Säuglinge nur dann, namentlich auch in der Zeit der Reparation, eine Hemmung im Längenwachstum aufwiesen, wenn die dargereichte Nahrung, wie z. B. die Frauenmilch, arm an Eiweiß- und Mineralstoffen war, während sie bei der eiweißreichen Eiweißmilch oder dem Larosan sehr schnell das Versäumte wiedereinholten. Neben der Fettarmut war es aber auch gerade der Eiweißmangel, der die Kriegskost als solche charakterisierte und sie insbesondere zum Aufbau des kindlichen Körpers so unzulänglich machte.

Bei meinen eingehenden Schüleruntersuchungen war im So m mer 1916, am Ende des zweiten Kriegsjahres, noch kein $Z$ urückbleiben 
im Längenwachstum hinter die an denselben Schulen gewonnenen Durchschnittszahlen aus der Friedenszeit festzustellen. Im Gegenteil, bei nicht wenigen Gruppen der Knaben aus den Volks-, Bürger- und Fortbildungsschulen fanden sich im 2 . Kriegsjahr größere Zahlen, durchschnittlich um $1-2 \mathrm{~cm}$ größere Längenzahlen als im Jahre 1913. Dieser Unterschied im 2. Kriegsjahr nach der Plusseite war um so auffallender, als sich gleichzeitig sehr deutlich und mit Sicherheit im Durchschnitt eine nicht gerade kleine Gewichtsabnahme feststellen ließ. Aber die Allgemeinheit der Kinder unterschied sich doch sehr und hatte in ihrem Äußeren kaum etwas gemein mit jenen disproportioniert gewachsenen Knaben aus der Streckungsperiode in der Pubertätszeit, die gleichfalls eine Längenzunahme bei Gewichtsstillstand, daneben aber auch noch mehr oder weniger andere Abnormitäten aufweisen (A ron).

Wesentlich anders fiel das Ergebnis der Längenmessungen an den Kindern ebenderselben Schulen im 3. Kriegsjahr, 1917, aus. Jetzt wurden fast durchweg in allen Altersstufen und in allen Gruppen kleinere Längenzahlen als in den vorangegangenen Jahren, auch als im letzten Friedensjahr ermittelt. Der Rückstand betrug 1917 gegenüber 1913 bei den Kindern aus der Volks- und Bürgerschule, in den unteren wie in den oberen Klassen, bis zu $2 \mathrm{~cm}$, etwas weniger bei den 14-17jährigen Lehrlingen. Gegenüber 1916 mit seinen größeren Durchschnittszahlen wurde vielfach ein Zurückbleiben um fast $3 \mathrm{~cm}$, festgestellt. Einige Jahrgänge, so das 6. und 14. Jahr, gerade Altersklassen mit ungünstigen konstitutionellen Verhältnissen, wiesen 1917 verhältnismäßig viele ausgesprochen kleine Kinder bzw junge Leute auf, was besonders deutlich auch bei der Ermittelung der Struktur der dadurch heruntergedrückten Durchschnittswerte zum Ausdruck kam. Aber in den meisten Jahrgängen bot der Aufbau der Durchschnittszahlen nichts Besonderes.

Schließlich 1918, im vierten Kriegsjahr, ermittelte ich im allgemeinen ähnliche Längenzahlen wie 1917. Eine weitere Vergrößerung des Rückstandes war kaum zu verzeichnen; vielleicht war der Unterschied gegenüber den Zahlen aus der Friedenszeit noch etwas regelmäßiger geworden. Gegenüber 1913 schwankte die Differenz in den einzelnen Altersstufen zwischen 0,8 und $2 \mathrm{~cm}$, in einzelnen Jahren, so $\mathrm{im} \mathrm{13.} \mathrm{Jahr,} \mathrm{betrug} \mathrm{sie} \mathrm{fast} 3 \mathrm{~cm}$ (siehe die Tabellen $\mathrm{S}$. 112--114).

An den Gymnasien und Realschulen, deren Schüler wesentlich vermögenden Familien und solchen aus dem Mittelstand angehörten, 
war sehr deutlich der Rückstand im Längenwachstum 1918 gegenüber 1913 größer und regelmäßiger als bei den Volkssehülern, wie aus den Tabellen leicht ersichtlich ist. Bei den herangewachsenen jungen Leuten war aber nach dieser Richtung der Unterschied im Rückstand nicht mehr so deutlich; sowohl die Lehrlinge wie die gleichaltrigen Gymnasiasten waren 1918 durchschnittlich $1-1 \frac{1}{2} \mathrm{~cm}$ kleiner als ihre Altersgenossen aus dem Jahre 1913.

Ǔbrigens blieb trotz dieser Verschiedenheit in der Größe der Hemmung bei den Kindern aus verschiedenem sozialem Milieu der Vorsprung, den die gut situtierten Kinder vor ihren minderbemittelten Altersgenossen in der Länge ganz allgemein und immer aufweisen, auch jetzt in der Kriegszeit, nur wenig verändert erhalten.

Ich untersuchte des weiteren, ob sich bzgl. dieser Wachstumshemmung ein Unterschied zwischen den gut konstituierten Kindern und den nur mittelmäßig entwickelten Knaben feststellen ließ. Die Zahl der mangelhaft entwickelten war zu klein, um zu diesem Vergleich herangezogen werden zu können. Alle Kinder waren auf Grund der Inspektion in 4 Gruppen eingeteilt, wovon unten (S.99) noch ausführlicher die Rede sein wird. Bei den nur mittelmäßig entwickelten Kindern war im allgemeinen, wenn auch nicht a usnahmslos der $R$ ückstand etwas größer und regelmäßiger als bei den gut konstituierten Knaben (S. 114, 115), wie eben überhaupt die schwächer entwickelten Individuen unter der Kriegsnot vielfach stärker litten als die von vornherein kräftig konstituierten Personen. Bei einer größeren Anzahl besonders schwächlicher, mangelhaft entwickelter Kinder, insbesondere bei hypoplastischen Individuen nach Toblers Bezeichnung, ließen die fortlaufenden individuellen Beobachtungen während der letzten Kriegsjahre ein außergewöhnlich langsames Längenwachstum erkennen, manchmal eine über ein ganzes Jahr sich hinziehende, fast einem Wachstumsstillstand gleichkommende Hem mung, Verzögerungen, wie ich sie in dieser Andauer sonst kaum beobachtete.

Bei den älteren Knaben, etwa bis zum 16. Jahr, trat die Hemmung des Längenwachstums im allgemeinen stärker in Erscheinung als bei den jüngeren Kindern. Im 6. Lebensjahr, wo meine regelmäßigen Messungen einsetzen, ist der Rückstand allenthalben schon sehr deutlich; er hat wohl schon sehr frühzeitig, vermutlich schon beim Säugling, eingesetzt. Uber ein Zurückstehen bereits der $\mathrm{Ne}$ ugebore nen nicht nur im Körpergewicht, sondern auch in der Längenentwicklung finde ich 
Angaben aus der Münchener Frauenklinik aus dem Jahre 1918 (Ma ro n) und 1919 (Binz).

Ich habe bei den Reihenuntersuchungen im Anschluß an die Längenmessung regelmäßig Bemerkungen bez. der Statur der Kinder gemacht, wenn sie gegenüber ihren Altersgenossen durch ihre Kleinheit oder durch ihre Größe und Schlankheit auffielen. Trotz der wenig scharfen Begrenzung dieser Begriffe lohnt sich ein Vergleich zwischen 1913 und 1918. Gerade in diesem letzten Jahr waren unter den Schulanfängern in der Volksschule, aber auch in einigen Vorschulklassen der höheren Schulen a uffallend viele a usges prochen kleine Kinder, fast dreimal soviel als in Friedensjahren (S. 119). Ich glaube nicht, daß es sich da um eine bloße Zufälligkeit handelte. Eine zweite Vermehrung auffallend kleiner Kinder ließ sich 1918 bei den 13jährigen feststellen. Schon unter normalen Verhältnissen steigt in diesen Jakren regelmäßig die Zahl der im Wachstum zurückbleibenden jungen Leute an, nämlich bei einem verspäteten Einsetzen des Pubertätsantriebs. Kinder von schwacher Konstitution wie auch solche aus einem sozial ungünstigen Milieu, aus unbemittelten Familien, werden verhältnismäßig oft davon betroffen. Die Erschwerung der Lebenshaltung in den letzten Kriegsjahren mochte noch häufiger die Verzögerung der Wachstumssteigerung in der Pubertät verursachen.

Ebenso deutlich, ja noch deutlicher ist aber auch der Unterschied zwischen 1913 und 1918 bei den nach der entgegengesetzten Richtung, durch a usgesprochene Größe und Schlankheit ausgezeichneten Knaben und jungen Leuten. Diese hochaufgeschossenen Knaben mit auffallend langen Extremitäten, schmaler langer Brust, bei verhältnismäßig geringem Massenwachstum, einem 'disproportionierten Längenwachstum, mit niedrigem Index ponderalis, finden sich nur selten in der Volksschule, fast nur im letzten Jahrgang etwas häufiger, auch nicht so häufig unter den minderbemittelten Fortbildungsschülern der breiten Volksschicht, um so häufiger aber in den höheren Schulen, hier schon rom 9. oder 10. Jahr ab, ganz besonders oft etwa im 13. Jahr.

In dem letzten Kriegsjahr war nun die Anzahl dieser a usgesprochen schlanken jungen Leuteganz a uffallend zurückgega ngen, derart, daß sie in manchen Altersklassen, z. B. vom 17. Jahr $a b$, fast ganz fehiten, aber auch unter den 11-13 jährigen lange nicht mehr so häufig waren wie sonst (S. 119). Pfaundler führt das frühzeitige In die Höhe-Schießen, dieses disproportionierte Längenwachstum mancher Großstadtkinder aus den bemittelten Familien 
auf eine U̇berkultur zurück, auf eine Utberernährung, auf Ưberfütterung, auf eine Utbertreibung sportlicher Betätigung, auf eine Ubertreibung der gesamten Gesundheitspflege. Der Wegfall oder auch schon die Erschwerung der Voraussetzungen zu solcher Lebensführung würde das Seltenerwerden des disproportionierten In die Höhe-Schießens erklärlich machen.

Nach allen unseren klinischen Erfahrungen über die aus äußeren Ursachen entstandenen Wachstumsstörungen ist auch von der vorliegenden Hemmung mit Sicherheit anzunehmen, daß es sich nur um eine vorübergehende, durchaus reparationsfähige Störung handelt, die unter einigermaßen günstigen Verhältnissen sogar sehr rasch wieder ausgeglichen sein wird. Am Ende meiner Beobachtungszeit besteht der Rückstand noch im 18. Lebensjahr fort; eher darf mit einer Verlängerung der Wachstumsperioden gerechnet werden, wofür reichliche Beobachtungen vorliegen (meine Arbeit, Zeitschr. f. Kinderheilk. 1. c.), als mit einem dauernd unausgeglichenen Rückstand.

Die ganze, hier beschriebene Wachstumsstörung ist auch nur als eine einfach $\mathrm{H}$ e m m ung zu betrachten; der Wachstumstypus, die Wachstumskurve erfuhr in ihrer Form keine wesentliche Änderung, abgesehen etwa von der $a b$ und zu zu beobachtenden geringen Verzögerung des Pubertätsantriebes; des Eintritts der Pubertätssteigerung.

Aus dem eben angeführten Grunde bestimmt zu erwartender Reparationsfähigkeit kommt der Tatsache der Hemmung des Längenwachstums der Kinder während der letzten Kriegsjahre auch mehr ein wissenschaftliches Interesse als eine praktische Bedeutung zu; sie ist ein Hinweis a uf die Intensität der allgemeinen Unterernäh rung der Kinder. Vielleicht entbehrt sogar diese Verlangsamung des Längenwachstums unter den gegebenen äußeren Verhältnissen nicht eines gewissen Vorteils, insofern als die dem Organismus zur Verfügung stehenden Wachstumskräfte wesentlich zum inneren Anbau und Ausbau verwendet und verbraucht wurden, nicht so sehr zum äußeren Aufbau, der zunächst weniger notwendig und wertvoll erscheint als jener.

\section{Der Gewichtsrückstand.}

Bei der Beurteilung der Unterschiede in den Gewichtszahlen gleichaltriger Knaben aus gleichem sozialem Milieu, im vorliegenden Falle aus denselben Schulen und Krippen, vor und während des Krieges, kommen namentlich vier Faktoren in Betracht: Unterschiede im Massenwachstum, in der Stärke der Muskelmasse, in der Größe des Fettan- 
satzes und Schwankungen im Wasseransatz. Die Veränderungen in der Stärke der Muskelmasse spielen bei den gesunden Kindern, um die es sich hier handelt, die kleinste Rolle, wenn auch da und dort die körperliche Arbeit und die sportliche Betätigung der älteren Schuljugend während der Kriegsjahre etwas umfangreicher gewesen sein mag. Bedeutsamer sind schon andauernde Verschiedenheiten im Wassergehalt des Organismus, indem die kohlehydratreiche Kriegskost den Wasseransatz in hohem Maße begünstigte. Doch ist gerade die Ödenkrankheit, der höchste Grad der Wasserretention, fast nur bei Erwachsenen, bei Kindern nur unter ganz besonderen Verhältnissen, in Anstalten, beobachtet worden. Auf bemerkenswerte Gewichtsschwankungen bei Schul- und Kleinkindern, die auf Änderungen im Wassergehalt des Körpers zurückzuführen waren, werde ich unten (S. 94) zu sprechen kommen.

Am meisten interessiert uns bei den vorliegenden Untersuchungen die Größe des Schwundes des Fettansatzes, als des Ausdruekes und Maßes der Unterernährung. Einen ungefähren Aufschluß hierüber gibt schon die Inspektion, besonders an den $Z$ wischenrippenräumen, einen besseren die Palpation, wobei sich leicht mehrere Grade der Abmagerung unterscheiden lassen (v. Pirquet). Aber genauere Bestimmungen lassen sich nur mittels der Wage erheben, unter Berücksichtigung der Tagesschwankungen und auch der Jahreszeiten. Und auch die Wägungen geben nur beim ausgewachsenen Individuum ein eindeutiges Resultat; beim Kind und Jugendlichen wird die Beurteilung des Ergebnisses der Wägungen vor allem durch die Gewichtszunahme beim Massenwachstum erschwert. Bis zu einem gewissen Grade von Genauigkeit läßt sich die Beteiligung des letzteren durch gleichzeitige Berücksichtigung der Änderung des Längenwachstums ermessen. Die Längenzunahme entspricht zum mindesten im allgemeinen auch einer Massenzunahme, trotz des in den Einzelheiten und namentlich auch zeitlich von einander verschiedenen und von einander unabhängigen Ablaufs des Längen- und Massenwachstums, beim schubweisen, alternierenden Aufbau und Ausbau des kindlichen Körpers. Schließlich ist auch noch das Vorkommen eines disproportionierten, eines einseitig gesteigerten Längenwachstums zu berücksichtigen, wie es während des Schulalters besonders zur Zeit der Pubertät fast physiologisch ist.

Ich habe bei meinen Untersuchungen die Ergebnisse der Längenmessungen und der Wägungen auf zweierlei Weise miteinander in Beziehung gebracht, einmal indem ich mein Beobachtungsmaterial außer nach dem Alter auch nach Gruppen von $5 \mathrm{zu} 5 \mathrm{~cm}$ Längenunter- 
schied ordnete und nun die Gruppen gleichaltriger, gleichgroßer Knaben zum Vergleich des Gewichts in den Jahren vor und während des Krieges einander gegenüberstellte. Außerdem habe ich, noch genauer und wissenschaftlicher, nach dem Vorgehen Pfa undlers, Arons u. a. die Längen- und Gewichtsmaße in dem Livischen Index ponderalis $100 \sqrt[3]{\text { Gewicht }}$

Lange miteinander in Beziehung gebracht und diese Zahl für viele Einzelindividuen, außerdem aber auch für die Durchschnittswerte von Länge und Gewicht aller Gruppen errechnet und miteinander verglichen.

Bei einer Gegenüberstellung der Wägungsergebnisse gleichaltriger Kinder und junger Leute aus demselben sozialen Milieu, aus ein- und denselben Anstalten bzw. Schulen in den Jahren 1913 und 1916, - zur Vermeidung von Fehlern wurden die Wägungen auch zu denselben Tagesund Jahreszeiten vorgenommen, - zeigen die Säuglinge, die Kleinkinder und auch noch die jüngeren Schulkinder bis zum 9. Lebensjahr durchschnittlich dasselbe Gewicht vor dem Kriege und im 2. Kriegsjahr. Dagegen bleiben die Volksschüler der Mittelstufe bereits um etwa $1 / 2 \mathrm{~kg}$ hinter ihren gleichgestellten Altersgenossen aus der Friedenszeit zurück, die besser situierten Mit telschüler um ein volles Kilo, und bei der herangewachsenen Jugend im Alter von 14-17 Jahren, bei den Lehrlingen aus der Fortbildungsschule, beträgt der Unterschied $1 \frac{1}{1} / 2-2^{1 / 2} \mathrm{~kg}$.

Noch größer und vor allem auch noch regelmäßiger ist der Rückstand im Gewicht 1916 gegenüber 1913 bei gleichzeitiger Berücksichtigung der Körperlänge, die sich in dieser Periode teilweise im entgegengesetzten Sinne verschoben hat (S. 84), also bei einer Gegenüberstellung der gleichaltrigen und gleichgroßen Knaben. Da weisen im zweiten Kriegsjahr auch schon die siebenjährigen Knaben einen Rückstand von $1 / 2 \mathrm{~kg}$ auf; bei den älteren Knaben und jungen Leuten steigt der Unterschied auf 2-21/2 kg. Dieses Zurückbleiben des Gewichts darf und $m u B$ bei dem eben erwähnten Verhalten des Längenwachstums ganz auf Fettverlust, auf eine Verkleinerung des Fettansatzes des Körpers zurückgeführt werden. Die Säuglinge und die Kleinkinder hatten in jener Zeit noch nicht an Gewicht abgenommen; ihre Ernährung hatte damals auch noch kaum eine Einbuße erlitten.

Wesentlich anders im dritten Kriegsjahr 1917. Da weisen auch die zwei- und dreijährigen Klein kinder, wenn auch nicht regelmäßig, so doch in vielen Gruppen, durchschnittlich eine $R \ddot{u ̈ c k s t a ̈ n d i g k e i t}$ 
im Gewicht von $1 / 2-1 \mathrm{~kg}$ gegenüber der Friedenszeit auf, ja schon die Säuglinge im 1. und 2. Lebensvierteljahr wiegen durchschnittlich um $200 \mathrm{~g}$, jene im vierten Quartal um $500 \mathrm{~g}$ weniger als vor dem Krieg. Die vier- bis fünfjährigen Kinder zeigten wechselnde Verhältnisse. Die jüngeren Volksschüler wiegen auch nur, wie die Kleinkinder, $1 / 2$ bis $1 \mathrm{~kg}$ weniger als 1913 , aber die älteren $\mathrm{Knaben} 1-2 \mathrm{~kg}$, die Lehrlinge durchschnittlich 2-4, ausnahmsweise $5 \mathrm{~kg}$. Einige Jahrgänge, so das 10. und 11. Jahr, zeigten etwas günstigere Verhältnisse.

In diesem Jahr 1917 ist, im Gegensatz zu 1916, zum mindesten bei den Schulkindern vom 6. bis 12 . Lebensjahr, der weitere Gewichts rückstand nicht so sehr a uf einen weiteren Fettverlust als vielmehr in der Hauptsache a uf die Hemmung und Verlangsamung des Massenwachstums zurückzuführen; denn die gleichaltrigen Kinder von gleicher Größe wiegen 1917 fast durchweg mehr, und zwar um $1 / 2 \_1 \mathrm{~kg}$ mehr als im Jahre 1916. Erst bei den älteren Knaben und noch mehr bei den herangewachsenen jungen Leuten liegen auch bei einer solchen Gegenüberstellung die Verhältnisse 1917 meist ungünstiger als 1916. Hier ist es neben der Verlangsamung des Wachstums zu einem weiteren Schw und der Fettreserven des Körpers gekommen. Bei den jüngeren Kindern ist diese Abwärtsbewegung im wesentlichen zum Stillstand gekommen.

$\mathrm{Daß}$ auch bereits die Säuglinge einen merklichen Rückstand im Körpergewicht aufwiesen, ist um so bemerkenswerter, als ihre Ernährung, zum mindesten die der Brustkinder, 1917 noch nicht Not gelitten hatte. Meine Untersuchungen über die Stillfähigkeit der Mütter meines großen poliklinischen Materials ergab, ebenso wie die anderer Autoren (Steinhardt, Hess, Bachaver) günstige Resultate: in Jahre 1913 waren $77 \%$ der Säuglinge von ihrer Mutter gestillt worden, $191680 \%, 191781 \%$. Die durchsehnittliche Stilldauer betrug 1913 19 Wochen, 191623 Wochen, 191722 Wochen, eine erfreuliche Besserung, die um so mehr ins Gewicht fällt, als in jenen Jahren die Arbeit der Frauen außerhalb des Hauses einen so außerordentlichen Umfang angenommen hatte. Der erwähnte Rückstand im Gewicht der Säuglinge betrifft fast ganz die künstlich genährten Flaschenkinder.

Im vierten Kriegsjahr 1918 habe ich im allgemeinen dieselben Durchschnittslängen und Gewichtszahlen ermittelt wie im dritten Kriegsjahr. Abgesehen von den ältesten Jahrgängen, insbesondere bei den Lehrlingen, war eine weitere Vergrößerung des Gewichtsrückstandes kaum festzustellen. 
Bemerkenswert ist die Verschiedenheit der Größe des Rückstandes in den einzelnen Jahrgängen. - Die in großer Zahl sich über zwei und drei Jahre fortsetzenden Kurven allwöchentlicher Wägungen ,gesunder “ Kinder aus der Krippe geben Aufschluß über das Zustandeko m men des Gewichtsrüekstandes bei den Kleinkindern. Nicht etwa als ob sich im dritten Kriegsjahr die regelmäßige, an sich geringfügige, wöchentliche Zunahme der Kinder in den einzelnen Altersstufen gegenüber früher verkleinert hätte, vielmehr liegt der Grund in den Gewichtsschwankungen und Gewichtsabnahmen, wie sie bei jedem, auch dem gesündesten Kleinkind im Laufe der Monate gelegentlich leichter Ernährungsstörungen oder bei irgendeinem anderen $Z$ wischenfall die regelmäBige Zunahme unterbrechen ; diese Abnahmen sind jetzt augenscheinlich bei gleicher Intensität oder richtiger gesagt bei gleicher Geringfügigkeit der klinischen Erscheinungen stärker und vor allem auch nachhaltiger geworden. Die Erholung, insbesondere gerade die Reparation des Gewichtsverlustes nach solchen Störungen erfolgt jetzt deutlich langsamer als zu früheren Zeiten.

Naturgemäß wird der Gewichtsrückstand im allgemeinen mit zunehmendem Alter größer, entsprechend der normalen absoluten Größe des Fettansatzes; aber auch schon im 11. bis 13. Jahr ist er nicht mehr viel kleiner als im 16. Jahr.

Gegenüber dem Verlust, wie ihn fast durchweg die Erwachsenen erlitten haben, - durchschnittlich mindestens $20 \%$ ihres Körpergewichts; in Anstalten, deren Insassen mehr oder weniger ganz auf die rationierte Nahrung angewiesen waren, 30 bis $40 \%$, - ist der Verlust der Kinder und Jugendlichen im allgemeinen nur als mäßig zu bezeichnen, natur. gemäß, bei dem viel geringeren Umfang ihres Fettpolsters. Ich habe den Verlust durchschnittlich mit 6 bis $7 \%$ des Körpergewichts errechnet; bei den herangewachsenen jungen Leuten stieg er bis $9 \%$.

Eingehende vergleichende Untersuchungen bei Kindern aus den verschiedenen Bevölkerungsschichten ergaben (S. 112-114), daß die K in der des unteren Mittelstandes früher, frühzeitiger, schon im zweiten Kriegsjahr und stärker an Gewicht einbüßten, als die Kinder a us der breiten Volksmasse der Arbeiter. Später glich sich der quantitative Unterschied teilweise wieder aus; doch blieben z. B. die kaufmännischen Lehrlinge immer mehr hinter ihren Durchschnittszahlen aus der Friedenszeit zurück, als dies bei den unbemittelten Taglöhnerlehrlingen der Fall war. Den grö $B$ ten und regel mäBigsten Gewich tsrückstand zeigten 1918 gegenüber 1913 die 14 jährigen 
und älteren Schüler des Gymnasiums, das am meisten von Angehörigen der oberen Stände und des Mittelstandes besucht war. Günstiger lagen die Verhältnisse in den mittleren und oberen Klassen von Gymnasien und Oberrealschulen, die einen stärkeren Einschlag von Schülern vom flachen Lande hatten. An einigen höheren Schulen fiel in den Vorschulklassen gerade die Kleinheit des Rückstandes auf. An einer Hilfsschule für Schwachbegabte mit vielen armen Kindern war der Gewichtsrückstand in vielen Altersklassen nur unbedeutend, im Gegensatz zu dem verhältnismäßig großen Rückstand im Längenwachstum. Vielleicht war gerade in diesen Familien die Ernährung während des Krieges besser als vorher. Im übrigen stehen die errechneten Feststellungen im allgemeinen in gutem Einklang mit den alltäglichen Wahrnehmungen über die Lebenshaltung der verschiedenen Bevölkerungsschichten im Kriege. Das sei hier schon betont, daß die Rückstände im Körpergewicht keineswegs, wie man dies vielleicht erwarten könnte, regelmäßiger oder gar größer waren als die Rückständigkeit des Längenwachstums; vielmehr traf das Gegenteil hiervon zu. Davon wird unten (Seite 97) noch ausführlicher die Rede sein.

Wie verhielt sich die Gewichtsrückständigkeit bei den Kindern von verschiedener Konstitution, von verschiedener allgemeiner Körperentwicklung? Während die mittelmäßig konstituierten Knaben hinsichtlich der Längenentwicklung durchschnittlich deutlich stärker im Rückstand blieben als ihre gut entwickelten Altersgenossen, wie dies oben (Seite 85) dargelegt wurde, war bezüglich des Gewichts ein solcher Unterschied nicht deutlich; die schwächer entwickelten Kinder büßten im Laufe des Krieges im allgemeinen nicht mehr an Gewicht ein als die gut entwickelten (Tafel II), während sie in mancher anderen Hinsicht, z. B. in ihrer Widerstandskraft gegenüber Infektionen, von der Kriegsnotlage viel stärker ergriffen wurden als die letzteren. Man darf wohl annehmen, daß ihr Gewicht deshalb nicht so sehr in Rückstand blieb, weil sie vielfach von vornherein bei einem ihrem Entwicklungszustand entsprechenden, nur mäßigen Ernährungszustand nicht allzuviel Fett zu verlieren hatten.

Ich habe bei den Reihenuntersuchungen regelmäßig, wie hinsichtlich der Statur die auffallend kleinen und die ausgesprochen großen, schlanken, so auch bezüglich des Ernährungszustandes die ausgesprochen mageren Kinder besonders bezeichnet. Utber ihre Anzahl in den einzelnen Kriegsjahren soll hier berichtet werden, ohne damit bindende Schlüsse auf die Ernährungsverhältnisse zu ziehen; denn magere Kinder bedeutet 
noch keineswegs abgemagerte, unterernährte. Die Zahl der Kinder, die bei entblößtem Rumpf auf den Untersucher den Eindruck der Magerkeit machen, ist im Schulalter, namentlich in der Periode der Streckung des Körpers, schon in normalen Zeiten verhältnismäßig groß. Ich ermittelte in Friedenszeiten einen geringen Ernährungszustand bei den einzelnen Altersstufen in 8 bis $16 \%$, bei den älteren Jahrgängen deutlich größere Zahlen als bei den jüngeren Kindern, und was gerade für die vorliegenden Betrachtungen besonders bedeutsam ist, unter den gut und besser situierten Schülern der höheren Lehranstalten ein entschieden häufigeres Vorkommen der Magerkeit, als bei den minderbemittelten Volksschülern. Es handelt sich eben bei der Magerkeit vielfach um eine innere Assimilationsbehinderung, bei mehr als ausreichender Nahrungszufuhr, wobei Vererbung, Neuropathie und andere Konstitutionsanomalien eine mehr oder weniger große Rolle spielen.

So hat sich auch im Laufe des Krieges im Gegensatz zu dem Gang der Ernährungsverhältnisse die A nzahl der mageren Kinder nicht wesentlich geändert (S. 119), ganz im Gegensatz zu der Anzahl der kleinen und der schlanken, großen Kinder (S. 86). Insbesondere bei der breiten Masse der unbemittelten Volksschüler zeigen die Zahlen keine wesentlichen, einheitlichen Verschiebungen, im Jahre 1916 wohl eine geringe Steigerung gegenüber 1913, weiterhin 1917 wieder eine geringe Abnahme, nur bei den Lehrlingen nochmals eine geringe $\mathrm{Zu}$ nahme, schließlich 1918 fast dieselben Zahlen wie 1913. In der gehobenen Volksschule, bei den Kindern des unteren Mittelstandes, ist am Ende des Krieges eher eine Vermehrung der Zahl der mageren Kinder zu bemerken. Aber in den höheren Schulen ist der Unterschied nach dieser Richtung vor und am Ende des Krieges wiederum nur gering und vor allem gar nicht einheitlich. Ihre Zahl schwankt nach wie vor zwischen einem Fünftel und einem Achtel aller Schulkinder.

Deutlicher ist ein Unterschied, trotz der naturgemäßen Geringfügigkeit der.Differenzen zwischen einst und jetzt, bezüglich des entgegengesetzten Zustandes zu erkennen, bezüglich der Fettsucht, eines überreichlichen Ernährungszustandes. Ich habe früher ausgesprochene Fettsucht unter den Kindern der höheren Lehranstalten in $2 \%$, unter den Volkssehülern in $1 / 2 \%$ ermittelt. Am stärksten war immer die Zeit bei Beginn der Pubertät beteiligt. Jetzt, 1918, habe ich ausgesprochene Fettsucht in der ganzen Volkssehule überhaupt nicht mehr beobachtet; in den höheren Schulen war ihr Vorkommen auf wenige Fälle, gerade bei Beginn der Pubertät, im 12. und 13. Jahr, beschränkt. 
Anschließend an diese Ergebnisse der generalisierenden Sammelstatistik seien die Resultate der individualstatistischen Methode, der fortlaufenden Wägungen ein und derselben Kinder mitgeteilt. 500 Knaben einer Volksschule, einer Mittelschule und einer Hilfsschule wurden regelmäßig alle Vierteljahr gewogen. Bekanntlich geht die Gewichtszunahme der Kinder wie auch ihr Längenwachstum im Lauf des Jahres recht ungleichmäßig vor sich: während sie im Winter und in der ersten Hälfte des Frühjahrs etwa mittelgroß ist, erfolgt bei Be ginn des Sommers bei nicht wenigen Kindern eine mehr oder weniger beträchtliche Gewichtsabnahme, in der Hauptsache durch Wasserverlust, bei der reichlicheren Bewegung im Freien, die sich manchmal erst nach Monaten wieder ausgleicht. Dann, von August bis Oktober oder November, findet die weitaus lebhafteste und regelmäßigste Gewichtszunahme statt, vor allem durch Massenwachstum, aber auch, namentlich während der Ferienzeit, gleichzeitig durch Fettansatz, durch Mast. Lokale, bzw. in dem sozialen Milieu gelegene Verschiedenheiten spielen dabei eine nicht geringe Rolle.

Ich ermittelte an derselben Volksschule, an der ich während des Krieges die Wägungen vornahm, in den Jahren 1906-1909 durchschnittlich im ersten Vierteljahr $53 \%$ Gewichtszunahmen von $1 / 2-2 \mathrm{~kg}$, $35 \%$ ohne wesentliche Änderung $( \pm 0,3 \mathrm{~kg}), 12 \%$ Gewichtsabnahmen von $1 / 2-1 \mathrm{~kg}$, im zweiten Vierteljahr $51 \%$ Zunahmen gegenüber vollen $20 \%$ Gewichtsabnahmen, im dritten Vierteljahr aber wieder $75 \% \mathrm{Zu}$ nahmen (darunter $29 \%$ von $11 / 2 \mathrm{~kg}$ und darüber) gegenüber nur $4 \%$ Gewichtsabnahmen, meist von $1 / 2 \mathrm{~kg}$, im vierten Quartal $63 \% \mathrm{Zu}-$ nahmen, 7\% Abnahmen.

Das zweite Kriegsjahr war ausgezeichnet durch eine erhebliche Vermehrung der Zahl der Kinder mit Gewichtsabnahmen über $1 / 2 \mathrm{~kg}$ im ersten und zweiten Vierteljahr, $19 \mathrm{bzw} .31 \%$, während die Anzahl der Kinder mit Gewichtszunahme entsprechend herunterging, auf $45 \mathrm{bzw}$. $26 \%$, dafür zeigte aber das dritte Quartal um so günstigere Verhältnisse, in vollen $91 \%$ Gewichtszunahmen, nur in $2 \%$ eine Gewichtsabnahme, derart daß die vorangegangene Ungunst wieder so gut wie ganz ausgeglichen wurde. Man konnte in jenem Jahr bei den Gewichtsverlusten an eine besonders starke Beteiligung der Fettreserven, an eine Abmagerung denken, dies um so eher, als die Gewichtsverhältnisse ziemlich parallel gingen dem Gang der Ernährungsverhältnisse, zuerst einer deutlichen Verschlechterung, dann wieder 
einer Besserung derselben, und als ferner deutlich gerade von vornherein gut genährte Kinder eine stärkere Gewichtsabnahme aufwiesen, während magere Kinder seltener abnahmen.

Aber die Fortsetzung dieser vierteljährlichen Wägungen im dritten Kriegsjahr zeigte dieselben Gewichtsschwankungen zum mindesten im Sommer in noch sehr viel höherem Maße. Anfang Juli 1917 wiesen gegenüber dem Gewicht vom April desselben Jahres ganz a ußerordentlich viele Kinder eine mehr oder weniger starke Gewichtsabnahme a uf, viel mehr als 1916, noch viel mehr als zu normalen Friedenszeiten, nämlich $49 \%$, gegenüber $31 \% 1916$ und $20 \%$ 1909. In der Mittelschule mit Kindern aus geordneten häuslichen Verhältnissen hatten in dem gleichen Zeitraum fast ebenso viele, $45 \%$, abgenommen, in der Hilfsschule aber mit ihren vielen armen Kindern volle $81 \%$, gegenüber nur $2 \%$ Zunahmen und $17 \%$ Gewichtsstillstand. Zwei Drittel der KKnaben wurden durch diese Gewichtsabnahmen wieder vorübergehend auf den Stand vom Januar zurückgebracht, ein Siebentel auf den Standpunkt im Oktober, fast ein Viertel aber nahezu auf den Stand vom Juli 1916, also zurück um nahezu ein volles Jahr.

Diese fast allgemeine Gewichtsabnahme bei im übrigen gutem Allgemeinbefinden und ohne vorangegangene Erkrankungen konnte in der Hauptsache nur durch eine ungew c̈hnlich starke Wasserabgabe am Anfang des Sommers erklärt werden. Letztere hatte aber zur Voraussetzung, oder sie wurde doch zum mindesten erleichtert und begünstigt durch einen ungewöhnlich starken Wasseransatz im Organismus; wie er durch den Wasser- und Kohlehydratreichtum der Kriegskost, - sie bestand gerade damals im wesentlichen aus Suppen und Gemüse, viel weniger aus Teigwaren und Mehlspeisen, - möglich und bedingt war. Diese großen Gewichtsschwankungen sind eine Eigentümlichkeit der Gewichtskurven des Jahres 1917, die 1918 lange nicht in diesem Umfang beobachtet wurden. In dem dritten Quartal 1917 wurde wohl in der großen Mehrzahl der Fälle dieser Sommerverlust wieder ausgeglichen; immerhin blieb aber die Zunahme in dieser Periode 1917 hinter jener von 1916 und noch mehr hinter denen aus Friedenszeiten deutlich zurück. So kam es, daß von den jüngeren Kindern im Herbst 1917, normalerweise nach der Periode stärkster Zunahme, $12 \%$ nicht mehr wogen als im Januar zuvor, und von diesen wieder die Hälfte, $6 \%$ sogar nicht mehr als sie ein ganzes $\mathrm{Jahr}$ zuvor gewogen hatten. 
Fortlaufende, vierteljährliche Wägungen an denselben $300 \mathrm{Volksschülern} \mathrm{im} \mathrm{Alter} \mathrm{von} \mathrm{6-14} \mathrm{Jahren.}$

(Zahl in Prozenten der Gesamtzahl.)

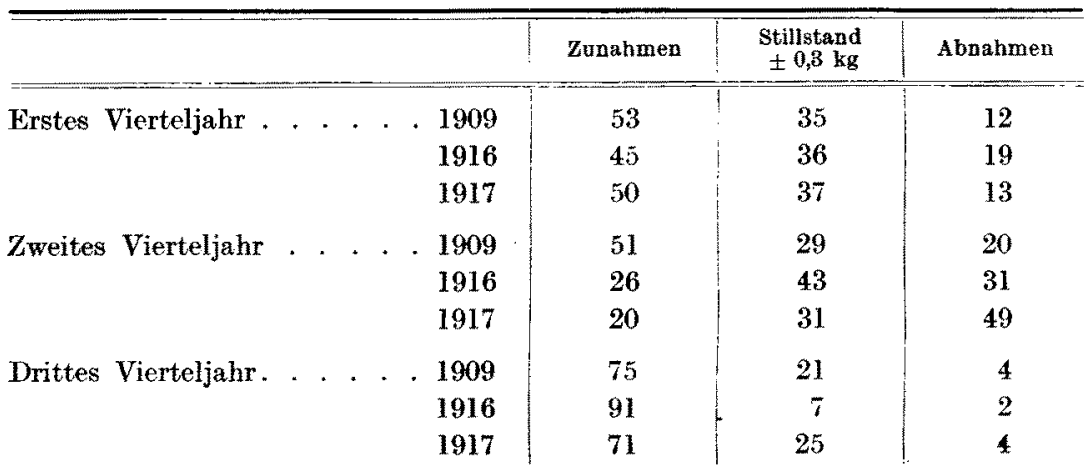

Der Index ponderalis.

In dem Bestreben, den bei den Messungen und Wägungen festgestellten Rückstand miteinander in Verbindung zu bringen, um die Hemmungen des Längen- und Massenwachstums in ihrer Bedeutung miteinander vergleichen zu können, benützte ich Livis Index ponderalis, den Pfaundler seinen, neue Gesichtspunkte eröffnenden Körpermaßstudien und A ron seinem Studium der Wachstumsstörungen zugrunde legten. All die einfacheren Formeln, wie das früher viel benutzte Zentimeter- oder Streckengewicht $=\frac{\text { Gewicht }}{\text { Länge }}$ oder die empirisch gefundenen Formeln, z. B. das Pignetsche Verfahren, der coéfficient de robusticité, oder die $\mathrm{O}$ p pen heimersche Formel, halten einer wissenschaftlichen Kritik ihres mathematischenAufbaues nicht stand (Pfa undler) und sind aufzugeben. Besonders empfehlenswert dürfte v. Pir quets Indexzahl sein, $\frac{\sqrt[3]{10 \cdot \text { Gewicht }}}{\text { Sitzhöhe }}$, da sie bei gleicher Entwicklung von Muskulatur und Fettpolster in allen Lebensaltern annähernd konstant bleibt und so zugleich ein gutes Maß für den Ermährungszustand abgibt. Die Formel kam für mich nicht in Betracht, da ich die Sitzhöhe, den Abstand der Sitzfläche von der Scheitelhöhe, nicht gemessen habe.

Livis Index ändert sich (jedenfalls mehr als der v. Pirquets) mit fortschreitendem Alter; entsprechend der Streckung des Rumpfes, besonders aber auch entsprechend dem starken Längenwachstum der 
Extremitäten im Laufe des Kindesalters, wird er vom Säuglingsalter ab fortschreitend kleiner bis etwa - bei Knaben- zum 14. oder 15. Jahr, um dann wieder deutlich rasch größer zu werden. Die Untersehiede von Jahr zu Jahr sind freilich nur klein, derart, daß es sich meist nur um ein oder zwei Zehntel handelt. Eine einfache Uberlegung sagt, daß bei Abmagerung der Index kleiner werden muß, ebenso bei großer Schlankheit, bei hoch aufschießenden Kindern mit einem im Verhältnis zur Körperlänge geringen Gewicht, bei dem disproportionierten Längenwachstum mancher Kinder in der Streckungsperiode ohne entsprechende Massenzunahme. Im Hinblick auf die vorliegenden Untersuchungen sei auch noch erwähnt, daß Pfa undler den Index bei den Kindern aus der Arbeiterklasse größer fand als bei den Kindern der Wohlhabenden, entsprechend einer im Verhältnis zur Körperlänge größeren Massenentwicklung bei den Kindern der breiten Volksmasse.

Bei einer Gegenüberstellung der nach Jahresklassen geordneten Indices gleicher Gruppen der Altersklassen ein und derselben Schule aus den Jahren 1913 und 1918 (S. 112-115) fällt sofort ein Cmstand auf: 1913 bilden die Indices fast immer, namentlich bei einigermaßen größerer Gruppenbildung, eine wohlgeordnete Reihe mit einem tiefsten Punkt im 14. Jahr, bei weniger gut situierten Knaben im 15. Jahr, bei ausgesucht gut entwickelten, gut situierten Knaben bereits im 12. Jahr. Die Reihe fällt nach diesem Minimum hin langsam ab und steigt dann rasch wieder an, derart, daß die Indices vom 17 . oder 18 . Jahr etwa denen vom 7. oder 6. Jahr entsprechen. 1918 dagegen weisen die entsprechenden Reihen nicht selten große Unregelmäßigkeiten auf, Schwa $n$ k ungen nach oben oder unten, kein deutliches Minimum, ähnlich wie sonst Reihen von ausgesucht nur mittelmäßig entwickelten Knaben. Man bekommt leicht den Eindruck, daß hier etwas Ungewöhnliches vorliegt.

Was nun die Werte selbst betrifft, so liegt es nahe, 1918 durchschnittlich kleinere Indices zu erwarten, infolge eines kleineren Zählers im Bruche, entsprechend dem Gewichtsrückstand. Dies trifft in der Tat zu bei der MehrzahlderGruppenderminderbemitteltenVolksschüler; hier überwiegt gewöhnlich von den beiden Rückständen, dem in der Längenentwicklung und dem Zurückbleiben in der Gewichtszunahme, der letztere. Nicht selten sind auch die Indices unverändert vor und während des Krieges; die Hemmung im Längen- und Massenwachstum halten sich die Wage. 
Deutlich anders liegen aber die Verhältnisse bei den besser und den gut situierten $\mathrm{Knaben}$ aus dem oberen Mittelstande und den vermögenden Ständen, in den Gymnasien und Realschulen. Hier sind die Indices in der großen Mehrzahl der Gruppen, in manchen Reihen aus Gymnasien fast ausnahmslos, 1918 größer als 1913, wenn auch oft nur um ein oder wenige Zehntel. Hier überwiegt die He m mung in der Längenentwicklung über die Rückständigkeit der Gewichtszunahme. Meist am bedeutendsten und auch am regelmäßigsten ist die Differenz bei den Knaben mit ausgesucht mittelmäßiger Konstitution. Bei ihnen steht die Störung des Längenwachstums besonders im Vordergrund, während der Gewichtsrückstand nicht so bedeutend ist. Ähnlich auch bei den kleinen Gruppen der schwachbegabten, körperlich, gesundheitlich wie auch sozial meist besonders ungünstig gestellten Hilfsschüler, die, wie oben schon erwähnt, vielfach einen bedeutenden Rückstand im Längenwachstum, kaum einen in der Gewichtszunahme aufweisen.

Die Beobachtung, daß die Indices gerade bei den gut situierten Knaben größer geworden sind, indem sie während des Krieges vor allem in der Längenentwicklung, verhältnismäBig weniger in der Gewichtszunahme zurückblieben, diese Beobachtung ist besonders interessant in Zusammenhang mit der oben erwähnten Einseitigkeit ihres Vorsprunges vor den minderbemittelten Altersgenossen, Dieser Vorsprung betrifft vor allem und überwiegend das Längenwachstum, weniger und in geringerem Maße das Massenwachstum. Pfa undler führt die ganze t’bermäßigkeit vieler Kinder der Reichen in der Großstadt auf eine künstliche Ubertreibung in der Frziehung und Pflege zurück, auf Uberernährung, auf eine Uberkultur. Die Beobachtung des Zurückbleibens im Wachstum während des Krieges gerade in der Richtung jenes Vorsprungs steht in gutem Einklang mit dieser Auffassung.

Konstitution und Konstitutionsanomalien.

In der folgenden Besprechung des Verhaltens der konstitutionellen Körperverfassung der Schulkinder und der herangewachsenen Jugend während des Krieges möchte ich den Begriff der Konstitution nicht in dem von Martius, Kraus, Tandler u.a. eng begrenzten Sinne als die Summe der rein ererbten, durch äußere Einflüsse nicht oder kaum veränderlichen Anlagen aufgefaßt wissen, sondern vielmehr in dem allgemeinen Sprachgebrauch der allgemeinen, körperlichen 
Verfassung, des allgemeinen Entwicklungszustandes, etwa der Konstitution plus der Disposition.

Im Anschluß an die Messungen der Länge und des Brustumfanges und an die Wägung habe ich jedes Kind in bezug auf seine ganze Erscheinung, die Körperbeschaffenheit, a uf Grund der Inspektion eingeschätzt, mit den Prädikaten gut, gut bis mittelmäßig, mittelmäßig und mangelhaft. Der durch lange Übung dieser Methode an demselben Material geschärfte Blick erfaßt und umfaßt gerade das nicht MeBbare kaum minder gut als Wage und Zentimetermaß das Meßbare. Freilich ist die Methode eine subjektive und macht Vergleiche oder die Zusammenfassung der Befunde verschiedener Untersucher mehr oder weniger unsicher; diesen Mißstand habe ich durch Verwendung ausschließlich eigener Beobachtungen vermieden. - Die das Gewicht und die Länge zusammenfassenden Formeln sind trotz ihrer Objektivität gerade als Konstitutionsmaß kaum zu verwerten; die Indices weichen wohl bei einem disproportionierten Wachstum oder bei erheblichen Änderungen des Ernährungszustandes mehr oder weniger von dem Durchschnittswert der betreffenden Altersstufe $a b$, gehen aber selbst bei großen konstitutionellen Verschiedenheiten ineinander über, wenn nicht gar durcheinander.

Die Gruppe der gut konstituierten Kinder umfaßt die gesund, frisch, leistungs- und widerstandsfähig erscheinenden Knaben. Unter den als mittelmäßig beurteilten befinden sich viele mit einem auch nur mittelmäßigen Ernährungszustand, vor allem aber die Kinder mit nur mäßig entwickelter Muskulatur, entsprechend schlaffer Haltung, nicht selten mit blasser Hautfarbe, all dies oft genug bei ausreichendem Skelettwachstum. Die praktisch belanglose $Z$ wischenstufe ,gut bis mittel mäßig" habe ich eingeschaltet, um gerade bei diesen wissenschaftlichen Untersuchungen die erste und dritte Gruppe durch Ausschaltung der fließenden Ubergänge gut voneinander zu trennen. In der vierten, nur recht kleinen Gruppe der mangelhaft entwickelten Kinder befinden sich namentlich die körperlich zurückgebliebenen, insbesondere die hypoplastischen Kinder der Toblerschen Nomenklatur.

Die nach diesen Gesichtspunkten durchgeführten Untersuchungen ließen im zweite $n$ Kriegsjahr 1916 bei der herangewachsenen Jugend, bei den Lehrlingen, noch keine Verschiedenheit gegenüber den Ergebnissen des Friedensjahres 1913 erkennen. Wohl aber war bei den Knaben im Schulalter, und zwar bei den minderbemittelten Volksschülern eine, wenn auch nur geringe Verschlechterung 
festzustellen, eine geringe Verschiebung der Prozentzahlen der einzelnen Konstitutionen nach der nächst weniger guten Rubrik; insbesondere wies die an sich nur kleine Zahl der mangelhaft entwickelten Kinder eine, wenn auch nur geringe Vermehrung auf. Anstatt eines oder zwei von ihnen fanden sich jetzt drei oder gar vier fast in jeder Klasse. Die schwächlichen Kinder waren es ja auch, welche bei der schärferen Rationierung der Nahrungsmittel zuerst nicht mehr auskamen und Not litten, was z. B. auch aus den Stoffwechselversuchen von Herbst hervorging. Die kräftigen, von Haus aus gesunden Konstitutionen wurden in ihrem Gesamtzustand seltener, weniger und auch erst später beeinträchtigt.

Im dritten Kriegsjahr, 1917, setzte sich diese Verschiebung nach der ungünstigen Richtung etwas fort, wohl auch wieder nicht in starkem Maße, auch nicht in allen Altersstufen, immerhin aber so, daß bei der Auszählung wieder eine geringe Verschlechterung nicht zu verkennen war. Am geringsten war der Unterschied bei den unteren Altersklassen. In den Mittel- und Oberstufen besonders auch der Mittelschule fiel namentlich die Verringerung der Anzahl der gut entwickelten Kinder auf. Die Zahl der schwächlichen, der mangelhaft entwickelten war jetzt auch bei der herangewachsenen Jugend etwas angestiegen. Unter ihnen waren auffallend viele tuberkuloseverdächtige; wie die Anzahl der Kinder und Jugendlichen mit manifester Tuberkulose, so war auch die Zahl der tuberkuloseverdächtigen bereits 1916, noch mehr 1917 bedeutend in die Höhe gegangen, eine Wahrnehmung, die in zahlreichen Veröffentlichungen wiederkehrt (z. B. bei Thiele).

Schließlich im vierten Kriegsjahr, 1918, war die Verschlechterung auch bei den Kleinkindern nicht zu verkennen; aber augenscheinlich ist gerade hier die fast durchgehende Verschiebung der Werte nach der nächst niederen Stufe, wie sie sich namentlich auch aus der individuellen, fortlaufenden Forschung ergab, nicht so oft eine Folge allgemeiner konstitutioneller Verschlechterung als vielmehr einer Häufung pathologischer Befunde, insbesondere von Konstitutionsanomalien, von denen nachher die Rede sein wird. Dagegen wiesen die jüngeren Schulkinder, sowohl die aus der Volksschule wie jene aus den Vorschulklassen der höheren Schulen, weiterhin im allgemeinen nicht mehr eine weitere Versohlechterung der Körperverfassung, der allgemeinen Entwicklung auf. Erst bei den etwas älteren Knąben, etwa vom 9 . oder 10 . bis zum 13. oder 14. Jahr, 
ist wieder fast durchweg, nahezu in allen Gruppen, eine mehr oder weniger deutliche Minderung festzustellen, besonders auffallend im 12. und 13. Jahr. Schließlich bei der herangewachsenen Jugend, etwa vom 15. Jahr ab, liegen die Verhältnisse wieder günstiger; hier ist seit 1917 nicht nur keine weitere Verschlechterung, sondern sogar wieder eher eine Besser ung eingetreten, derart, daß sich die errechneten $\mathrm{Zah}$ len denen aus den Friedensjahren nähern (S. 116-118).

Zusammenfassend läßt sich sagen, daß im Vergleich zu den Rückständen, die das Längenwachstum und die Gewichtszunahme aufweisen, die Veränderungen im konstitutionellen Verhalten, in der allgemeinen Entwicklung der Kinder, im allgemeinen nicht so bedeutend, oft nur geringfügig sind. Das aber liegt ja auch im Wesen der Konstitution begründet, die Beständigkeit, ein in seinem Wesen nicht umgestaltbarer, durch die Lebensweise höchstens leicht modifizierbarer Zustand (Hip po k rates). Diese Feststellung entsprach auch im allgemeinen dem Aussehen der Kinder; wenn man sie in der Krippe oder im Hort, im Schulzimmer oder auch auf der Straße, bei ernster Arbeit oder im ausgelassenen Spiel betrachtete, war der Eindruck selbst im letzten Kriegsjahr nicht so sehr verschieden von dem gewohnten Anblick in Friedenszeiten. Freilich hatte sich das Aussehen der Kinder auch nur sehr langsam verändert, nicht wie bei den Erwachsenen ziemlich rasch im Jahre 1916. Als ich Gelegenheit hatte, im Frühjahr 1919 eine größere Anzahl dieser Kinder ,herausgefüttert" wiederzusehen, da war der Unterschied gegenüber 1918 doch recht deutlich.

Von den verschiedenen Konstitutionsanomalien und ähnlichen Zuständen sei zunächst der häufigste anormale Befund bei den Schulkindern besprochen, die ,Blutarmut", ein Befund, den die Schulärzte bis zu 30, ja $40 \%$ bei ihren Schulkindern feststellen, eine blasse Hautfarbe der Kinder, insbesondere eine blasse Gesichtsfarbe, im Verein mit mehr oder weniger deutlichen anderen Erscheinungen, wie Kopfschmerz, Mattigkeit, kühlen Extremitäten. Das Wesen und die Bedeutung dieser Anämie ist noch keineswegs geklärt, nur das eine steht sicher, daß es sich in der großen Mehrzahl der Fälle, auch dort wo die Blässe der äußeren Haut vergesellschaftet ist mit mehr oder minder starker Blässe der sichtbaren Schleimhäute, es sich nur um eine Schein an ämie handelt, indem die Blutuntersuchung keine merkliche Herab- 
setzung des Hämoglobingehalts ergibt; 70-75\% Hämoglobin nach Sahli liegen im Kindesalter noch in der physiologischen Breite. Ebensowenig ergibt sich aus dem klinischen Befund ein Grund zur Annahme einer Oligämie, einer Herabsetzung der Gesamtblutmenge. Vielmehr liegt bei dieser Anämie, wie auch bei der sog. Schulanämie, der während und durch den Schulbesuch ausgelösten Anämie, im wesentlichen eine ungenügende Füllung gewisser Abschnitte des Gefäßsystems vor, wofür z. B. Erich Müller als Ursachen anführt: 1. eine neuropathische Konstitution mit labilem Gefäßsystem, das gerade durch die Schulanstrengungen bei hypersensitiven Kindern in einen allgemeinen Reizzustand versetzt wurde, 2. Störungen in den Unterleibsorganen und dadurch bedingte abnorme Verteilung des Blutes im Körper, 3. eine infektiös-toxische Basis, nach Erkrankungen an Grippe mit anhaltendem Fieber oder bei Erkrankungen des lymphatischen Gewebes (Tuberkulose, Syphilis). A ron legt bei den schulanämischen Typen Nachdruck auf ein disproportioniertes, beschleunigtes Längenwachstum, auf eine durch Verlängerung des Gefäßsystems bedingte schlechte Blutfülle peripherer Gefäßpartien. Czerny erinnert an die Bedeutung der Wärmestauung im Schulzimmer.

Was nun die Häufigkeit dieser Anämien bei den Schulkindern während des Krieges anbelangt, so seien in erster Reihe die Ergebnisse wiederholter Blutuntersuchungen an 1600 Schulkindern aus Leipzig und Umgebung von Me yrich angeführt, der mit der Dauer des Krieges, trotz der verschiedenen Ernährungsschwierigkeiten, sowohl bei den Volksschülern wie bei den Fortbildungsschülern, in allen Altersstufen, ein fortgesetztes Steigen des Hämoglobingehalts feststellte, so bei 9 -10jährigen Knaben von durchschnittlich $69 \%$ Hämoglobin im April 1915 auf $70 \%$ im April 1916, bei Mädehen von $75 \%$ im November 1915 auf $78 \%$ im April 1916. Höhere Grade von Oligochromämie und Oligocythämie fanden sich im späteren Verlauf des Krieges überhaupt nicht mehr.

Ich stellte durch genaues Auszählen der Kinder und jungen Leute mit blassem Aussehen und blassen Schleimhäuten im 3. Kriegsjahr bei den Lehrlingen eine $Z$ unahme fest von 9 a uf $13 \%$, bei den unbemittelten Volksschülern eine mäßige Abnahme, bei den Kindern des Mittelstandes eine ebenso große $Z$ unahme. Im 4. Kriegsjahr war die Zahl der Blutarmut in der Volksschule wie in der Mittelschule deutlich kleiner geworden, so daß jetzt hier weniger Blutarme gefunden wurden als in Friedens- 
zeit, durchschnittlich $10 \%$ gegenüber $14 \%$ früher. Eine noch deutlichere Besserung war in nahezu allen höheren Schulen festzustellen. In den Vorschulklassen, bei den 6-9jährigen Kindern, war der Unterschied noch am geringsten, um so größer aber bei den 10-15 jährigen Knaben, wo in einigen Klassen die Zahl der Blutarmen bis auf die Hälfte früherer Jahre zurückging.

Blutarmut (Zahl der Knaben in Prozenten).

\begin{tabular}{|c|c|c|c|c|c|c|c|c|c|c|c|c|}
\hline \multirow{2}{*}{ Aiter } & \multicolumn{2}{|c|}{ Gymnas. I } & \multicolumn{2}{|c|}{ Gymbas. II } & \multicolumn{2}{|c|}{ Gymnas. III } & \multicolumn{2}{|c|}{$\begin{array}{l}\text { Oberreal- } \\
\text { sehule }\end{array}$} & \multicolumn{2}{|c|}{ Mittelschule } & \multicolumn{2}{|c|}{ Volkssehule } \\
\hline & 1913 & 1918 & 1913 & 1918 & 1913 & 1918 & 1913 & 1918 & 1913 & 1918 & 1913 & 1918 \\
\hline $6-9$ & 16 & 14 & 8 & 8 & 11 & 8 & 20 & 10 & 10 & 19 & 17 & 6 \\
\hline $10-12$ & 14 & 9 & 12 & 9 & 13 & 17 & 18 & 11 & 9 & 10 & 12 & 11 \\
\hline $13-15$ & 15 & 7 & 12 & 4 & 6 & 13 & 17 & 9 & 13 & 17 & 20 & 8 \\
\hline $16-18$ & 10 & 6 & 3 & 5 & 2 & 2 & 7 & 4 & & & & \\
\hline
\end{tabular}

Ich habe einige leicht faßbare Gruppen dieser blassen Schulkinder, die aber in der Gesamtheit doch nur einen Bruchteil aller blutarmen Kinder ausmachen, ausgesondert und 1913 und 1918 einander gegenübergestellt. Die ausgesprochenen $\mathbf{N}$ e uropathen unterden blassen Kindern haben im Laufe des Krieges noch am stärksten abgenommen (von 20 auf $8,5 \%$ ), nicht viel weniger aber auch der Typ der hocha ufgeschossenen, schlanken, blassen Knaben (11:6\% nur kleine Zahl der blassen, t uberkuloseverdächtigen Kinder mit stärkeren Schwellungen der Halsdrüsen oder mit Resten früherer Skrofulose u. a. ist deutlich größer geworden. Auch die absolut sehr viel größere Zahl der konstit utionell blutarmen Kinder mit mäßig entwickeltem Körperbau, mit mäßigem Ernährungszustand, Magerkeit und gering entwickelter Muskulatur hat zugenommen von 3 auf $4 \%$, während der noch größere Rest der übrigen blassen Kinder ohne weitere Besonderheiten und Anomalien in demselben Maße kleiner geworden ist $(5,1: 4,3 \%)$.

Im frühen Kindesalter, bei den Kleinkindern, spielt der Häufigkeit nach die alimentäre A nämie die Hauptrolle, eine echte Anämie mit mehr oder weniger starker Herabsetzung des Hämoglobingehalts des Blutes bei gewöhnlich nur unbedeutender Verminderung der Zahl der Erythrocyten. Sie ist auf den schädigenden Einfluß einer allzu reichlichen Milchnahrung, insbesondere des Milchfettes, zurückzuführen, und bei dieser Entstehungsweise ist es leicht erklärlich, daß auch diese Form der Anämie, namentlich die Zahl der wohlgenährten Kleinkinder mit 
dem auffallend weißen, fast gelblichen Teint, bedeutend seltener geworden ist.

Ausgesprochen günstig liegen die Verhältnisse während des Krieges bei den Haltungsanomalien und Verkrümmungen der Wirbelsäule. Ich trenne die schwereren, rachitischen Verkrümmungen der Wirbelsäule, deren Entstehung aus der frühen Kindheit datiert, die in nicht kleiner Zahl in die Schule, insbesondere in die Volksschule, mitgebracht werden, um sich weiterhin nicht zu vermehren, nur zu verschlimmern, und die sich vor allem durch die Steifheit und Starre des ganzen Skeletts klinisch von der anderen Form unterscheiden, von den ko nstitutionellen Verkrümmungen (Schulthess), Hoffas, Langes habituellen Skoliosen, Schanz' Wachstumsskoliosen, durch statische Ủberinanspruchnahme der Wirbelsäule. Diese Haltungsanomalien sind bedingt durch eine gewisse Weichheit, eine verminderte Widerstandskraft des ganzen Skeletts, seiner Bänder, durch Muskelschwäche, insbesondere einer Schwäche der Rückenmuskeln, wobei bald das Skelett, bald die Muskeln einen größeren Anteil an der Deformität haben. Sie setzen erst im 8. oder 9. Lebensjahr ein, vermehren sich von Klasse zu Klasse bis ins jugendliche Alter, indem bei diesen Rückenschwächlingen - hierin liegt die eigentliche Ursache, - das stundenlange Stillsitzen in der Schulbank, eine gewohnheitsmäßige Schiefhaltung namentlich beim Schreiben, das Verharren in zusammengesunkener Sitzhaltung die Anomalie auslöst und sie vor allem auch weiterhin verschlimmert. Ihre Domäne sind die höheren Schulen, insbesondere die Knabenschulen. Bald handelt es sich um eine Skoliose, bei den älteren Schülern vor allem um die rechtskonvexe Totalskoliose mit Hochstand der rechten Schulter, oder mit Vertiefung des einen, Verflachung des anderen Taillendreiecks, bald um den runden, hohlen, oder hohlrunden Rücken in allen Abstufungen, namentlich mit hochsitzender Kuppe, all dies immer bei großer Beweglichkeit der Gelenke, bei einer großen Fähigkeit derselben zu Úberstreckungen, nicht immer bei deutlicher Schlaffheit der Extremitätenmuskulatur.

Im Laufe der Kriegsjahresind nun alle diese Deformitäten der Wirbelsäule bei den Schulkindern in einem sehr deutlichen Maße seltener geworden, alle mit Ausnahme der an sich sehr kleinen Gruppe der hochgradigen fixierten Kyphoskoliosen mit mehr oder minder starkem Rippenbuckel, die meist auf schwerste Rachitis in den ersten Lebensjahren zurückzuführen sind, das Schmerzenskind der Orthopäden. Aber schon die mit großer Wahrscheinlichkeit, 
bei anderen Resterscheinungen der englischen Krankheit, als rachitisch anzusprechenden geringfügigen fixierten Skoliosen zweiten Grades, namentlich unbemittelter Volksschüler, sind deutlich seltener geworden, noch mehr die dorsoventralen Haltungsanomalien der letzteren. Noch deutlicher ist aber die Besserung in den höheren Schulen, die oben als die Domäne der konstitutionellen, habituellen Verkrümmungen bezeichnet wurden. Hier nahm bei meinem Material in früheren Jahren die Anzahl der Kinder mit schlaffem Rundrücken von $3-4 \%$ in den Vorschulklassen bis $7-10 \%$ im 15. bis 18. Jahre zu; jetzt steigt deren Zahl von $2-3 \%$ auf $6-7 \%$ im 14. bis 16 . Jahr an. Im 17. und 18. Lebensjahr ist ihre Anzahl 1918 auffallend klein.

Haltungsanomalien und Verkrümmungen der Wirbelsäule.

(Anzahl der. Knaben in Prozenten.)

\begin{tabular}{|c|c|c|c|c|c|c|c|c|}
\hline \multicolumn{5}{|c|}{ Höhere Schulen } & \multicolumn{4}{|c|}{ Volks- und Mittelschulen } \\
\hline \multirow[b]{2}{*}{ Alter } & \multicolumn{2}{|c|}{1913} & \multicolumn{2}{|c|}{1918} & \multicolumn{2}{|c|}{1913} & \multicolumn{2}{|c|}{1918} \\
\hline & $\begin{array}{l}\text { Sko- } \\
\text { liosen }\end{array}$ & $\begin{array}{c}\text { Rund- } \\
\text { rücken }\end{array}$ & $\begin{array}{l}\text { Sko- } \\
\text { liosen }\end{array}$ & $\begin{array}{c}\text { Rund- } \\
\text { rücken }\end{array}$ & Skoliosen & $\begin{array}{l}\text { Rund- } \\
\text { rücken }\end{array}$ & Skoliosen & $\begin{array}{c}\text { Rund- } \\
\text { rücken }\end{array}$ \\
\hline 7 & 6 & 3 & 3 & 4 & 8 & - & - & 3 \\
\hline 8 & 11 & 4 & 3 & 2 & 10 & 5 & - & - \\
\hline 9 & 8 & 3 & 7 & 4 & 9 & 6 & 7 & 1 \\
\hline 10 & 11 & 5 & 6 & 3 & 10 & 1 & 3 & 1 \\
\hline 11 & 12 & 4 & 6 & 2 & 10 & 2 & 5 & 4 \\
\hline 12 & 16 & 3 & 6 & 4 & 13 & 1 & 11 & - \\
\hline 13 & 21 & 4 & 7 & 4 & 15 & 1 & 5 & - \\
\hline 14 & 16 & 6 & 5 & 9 & 17 & 5 & - & 3 \\
\hline 15 & 18 & 7 & 10 & 6 & - & - & - & - \\
\hline 16 & 24 & 10 & 6 & 7 & 一 & - & - & - \\
\hline 17 & 21 & 7 & 6 & 2 & 一 & - & - & - \\
\hline 18 & 24 & 7 & 3 & 4 & 一 & - & - & - \\
\hline
\end{tabular}

Am a uffallendsten ist aber der Unterschied in allen Altersklassen der Gymnasiasten und Realschüler hinsichtlich der seitlichen Verbiegungen 1. und 2. Grades. Früher, 1913, eine wenn auch unregelmäßig fortschreitende Vermehrung von 8 bis auf $24 \%$, vom 6 . bis 18. Lebenjahr; jetzt, 1918, in den Vorschulklassen nur 3\%, in den Mittelund Oberklassen nur 6\%, nur einmal, im 15. Jahr, ausnahmsweise $10 \%$ Skoliosen. Die leichtesten Grade sind verhältnismäßig noch seltener geworden als die Skoliosen zweiten Grades, die sich erst bei Schwebehang wieder mehr oder weniger ausgleichen. 
Der skizzierte Unterschied in der Häufigkeit der osteopathischfunktionellen Skoliosen ist während des Krieges größer, als or in dieser oder auch in der entgegengesetzten Richtung bei irgendeiner anderen Anomalie oder bezüglich eines Wachtumsbefundes zu beobachten gewesen wäre. Die Ursache dieser bemerkenswerten Verbesserung, die noch um so auffallender ist, als die Unterernährung, die Verschlechterung der häuslichen Verhältnisse und die, wenn auch nur geringfügige Verschlechterung im allgemeinen konstitutionellen Verhalten, in der Gesamtentwicklung, im entgegengesetzten Sinne wirken mußte, ist nicht schwer zu finden; sie liegt in der außerordentlich starken Verkürzung der Unterrichtszeit, des Schulbesuchs. Fast während der ganzen Kriegsdauer war in allen Schulen die tägliche Unterrichtszeit auf die Hälfte herabgesetzt; dazu kamen noch die vielen freien Tage für Sammlungen a. a., kam noch die Verlängerung der Ferien usw. Dem Schulbesuch, und zwar dem lange anhaltenden, schlieBlich ermüdend wirkenden Schulbesuch mit seinen $Z$ wangshaltungen, oder auch schon dem ruhigen Stillsitzen in der Schulbank kommt aber ein wesentlicher EinfluB beim Zustandekommen der konstitutionellen, osteopathischfunktionellen Haltungsanomalien jeder Art zu. Wohl ist der Begriff einer spezifischen, habituellen Schulskoliose aufgegeben worden, weil als das Wesentliche und Grundlegende die konstitutionell verminderte Widerstandskraft des Skeletts, des Bandapparats, die konstitutionelle Schwäche der Rumpfmuskulatur erkannt wurde. Aber neben dieser Disposition kommt der gewohnheitsmäBig schlechten, schiefen Haltung in der Schulbank, inbesondere beim Schreiben, kommt dem stundenlangen Verharren in zusammengesunkener Haltung, kommt überhaupt der Beschränkung freier Bewegung eine wesentliche, auslösende, mitwirkende, verstärkende Rolle beim Zustandekommen der Kyphosen und Skoliosen während der Schulzeit zu, und so ist die fast einzig dastehende deutliche Verbesserung gerade dieser Verhältnissse während des Krieges vollkommen erklärlich.

Nicht so einheitlich wie bezüglich der Haltungsanomalien und der Anämie der Schulkinder liegen die Verhältnisse hinsichtlich der neuropathischen Konstitutionsanomalie und deren Manifestationen. Sie ist bei den schulärztlichen Untersuchungen auch schon nicht so leicht erfaßbar wie jene, wenngleich die Lehrpersonen wertvolle Hinweise geben können. Abgesehen von dem ganzen Verhalten des Kindes bei der Untersuchung, einem aufgeregten, schreckhaften Wesen oder motorischer Unruhe, Zuckungen, und neben dem Verhalten der Reflexe 
gibt besonders der Puls und die Herztätigkeit wichtige Aufschlüsse, so eine auffallende, vorübergehende Pulsbeschleunigung oder die Unregelmäßigkeit des Pulses, noch mehr eine vorübergehende Verstärkung der Herztätigkeit, ferner leicht sichtbare vasomotorische Besonderheiten, Dermographie u. a. mehr.

Um eine $\mathrm{Zu}$ - oder Abnahme der Erscheinungen der neuropathischen Konstitutionsanomalie im Schulalter feststellen zu können, muß man sich vor allem an die höheren Schulen wenden, wo sie in weit höherem Maße zu Hause ist als in der Volksschule. Ich fand sie in den einzelnen Gymnasien und Oberrealschulen im 4.Kriegsjahr bald häufiger, bald seltener als zu Friedenszeiten. Am häufigsten war folgendes Verhalten: bei den 6-12jährigen Knaben 1918 gegenüber 1913 bald mehr, bald weniger deutlich ein selteneres Vorkommen, dagegen bei den älteren Kindern und bei den herangewachsenen jungen Leuten vom 13, bis 18 . Jahr eine ebenso de utliche $Z$ unahme. Als Beispiele seien folgende Zahlen angeführt:

Neuropathische Konstitution.

(Alle Zahlen in Prozenten.)

\begin{tabular}{|c|c|c|c|c|c|c|}
\hline \multirow{2}{*}{ Alter } & \multicolumn{2}{|c|}{$\begin{array}{l}\text { Gymnasium (gut situierte } \\
\text { Kinder) }\end{array}$} & \multicolumn{2}{|c|}{$\begin{array}{c}\text { Gymasium (Mittel- } \\
\text { stand) }\end{array}$} & \multicolumn{2}{|c|}{ Mittelschnle } \\
\hline & 1913 & 1918 & 1913 & 1918 & 1913 & 1918 \\
\hline $6-9$ & 10 & 6 & 10 & 8 & & \\
\hline $10-12$ & 8 & 6 & 10 & 5 & 4 & 3 \\
\hline $13-15$ & 11 & 14 & 7 & 7 & 5 & 2 \\
\hline $16-18$ & 12 & 16 & 12 & 12 & 6 & 7 \\
\hline
\end{tabular}

Wichtiger als diese in Zahlen zum Ausdruck kommenden Verschiebungen ist die Wahrnehmung, daß die verschiedenen Erscheinungen der Neuropathie bei den Untersuchungen im Jahre 1918 viel intensiver in die Erscheinung traten als früher. Zahlreichen Schülern merkte man ihre starke Erregung, ihre innere und äußere Unruhe, ihre Ubererregbarkeit ohne weiteres an. Nun war freilich gerade in jener Zeit mein Wirkungskreis außerordentlich oft, bei Tag und Nacht, durch Fliegeralarme und Fliegerabwehrkämpfe beunruhigt, und die klinische Beobachtung und noch mehr die hausärztliche Praxis haben reichlich gezeigt, bis zu welchen Graden hochgradiger Ubererregbarkeit solche Kriegsereignisse gerade bei neuropathisch veranlagten Kindern oder bei solchen aus nervösen Familien, aus einer nervösen Umgebung, 
führen können. Aber auch abgesehen von diesen Zuständen hat sich die Psyche des Kindes und ganz besonders die des neuropathischen Kindes im Laufe der Kriegsjahre merklich geändert, was auch auf die rein neuropathischen Erscheinungen nicht ohne Einfluß sein konnte.

$\mathrm{Zu}$ Anfang des Krieges allenthalben Lebendigkeit, Frische, ein Plus von Energie und Arbeitsfähigkeit. Das haben sich aber während der vier Jahre nur die körperlich und geistig kräftigen Naturen bewahrt. Bei den schwächer veranlagten Kindern machte sich mehr und mehr eine gewisse Schlaffheit, namentlich 1918 eine auffallend rasche Ermüdbarkeit, Zerstreutheit, Gleichgültigkeit bemerkbar, dazu in den Mittelstufen eine mangelnde Auffassungskraft, bei den höheren Schülern ein Erlahmen des Pflichtgefühls, der Willenskraft. Daneben war aber auch bei vielen Kindern aller Altersstufen und ganz besonders bei den jüngsten eine außerordentliche Empfindsamkeit, ein sensitiv gesteigertes Wesen, eben eine dauemde Ubererregbarkeit wahrzunehmen, die sich dann bei den Neuropathen in besonders hohem Maße äußerte. Aber auch jetzt noch unterschied sich die Veränderung beim psychoneuropathischen Kinde von der des psychisch schwachen Erwachsenen. Dort im wesentlichen Apathie, Gleichgültigkeit oder Úberempfindlichkeit, nur in Ausnahmefällen Hang zu Eigentums- und anderen Vergehen, hier vor allem eine psychische Depression, ein kummervolles Gedrücktsein und dadurch eine Leistungsunfähigkeit.

Wie schon angedeutet, ist es nicht angängig und wäre es verkehrt, diese Änderung der geistigen Verfassung beim Kinde allein auf die Unteremährung zurückzuführen. Da kamen ebensosehr in Betracht die veränderten häuslichen Verhältnisse, die unzureichende Einwirkung des Elternhauses bei der Abwesenheit des Vaters, vollends gar bei der Mutterlosigkeit, wenn diese dem Verdienst außer dem Hause nachging und die Kinder sich selbst, der Straße überließ, dazu die wechselnden Eindrücke der Straße, welche das Kind in nachhaltigerer Weise beeinflußten als der stark verkürzte Unterricht, endlich die langanhaltenden, nachhaltigen Nervenerregungen durch die mannigfachen Kriegsereignisse; all diese Veränderungen der Umwelt, wie sollte das an dem leicht beeinflußbaren Nervensystem des Kindes spurlos vorübergehen!

Bezüglich neuropathischer Zustände im frühen Kindesalter glaubte Kettner schon 1915 eine Vermehrung der Tetanie bei Säuglingen der ersten Lebensmonate, ja schon der Neugeborenen gefunden zu haben; 
er hielt dies für einen Ausdruck der nervösen Beeinflussung der Mütter während der Schwangerschaft. Es konnte aber von keiner Seite diese Beobachtung bestätigt werden.

Während das Untersuchungsmaterial aus den Schulen, den Volksschulen wie den höheren Schulen, sich vorzüglich eignet zu Studien über die bisher besprochenen Konstitutionsanomalien, über deren Verhalten und Hänfigkeit während des Krieges, sind die Krippen und Horte und die Kinderpoliklinik die wesentliche Stelle zu Untersuchungen über das Verhalten der Rachitis während der Kriegsjahre. Die hierüber an einem großen Material ermittelten Zahlen entsprachen im zweiten Kriegsjahr noch dem Durchschnitt aus Friedenszeiten, im dritten Kriegsjahr gingen sie bereits etwas darüber hinaus: ich zählte stärkere Grade von Rachitis bei denselben Altersstufen durchschnittlich 1917 in $23 \%, 1913$ in $20 \%$. Zum guten Teil mochte diese Vermehrung auf den längen, strengen Nachwinter und den dadurch verkürzten Aufenthalt der Kinder im Freien zurückzuführen sein. Im Frühjahr 1918 waren aber die Zahlen schwerer Rachitis bei den meisten Altersklassen des Kleinkindes noch größer, wieder um mehrere Prozent, entsprechend dem Maße, in dem sich weiterhin die gesamte $\mathrm{Hygiene}$ des $\mathrm{K}$ leinkindes und ganz besonders diejenige des in Pflege gegebenen Kleinkindes verschlechtert hatte.

Im Gegensatz hierzu fand ich 1918 bei den etwas älteren Kindern in den unteren Klassen der Volksschule die Zeichen inveterierter Rachitis etwas seltener als 1913, jetzt in 9 , früher in $12 \%$, in den Vorschulklassen der höheren Schulen nach wie vor in etwa $3 \%$. Für die vorliegenden Untersuchungen sind aber diese Befunde ziemlich belanglos, da in diesen Fällen die Entstehung und der Höhepunkt der Rachitis noch in eine Zeit vor dem Kriege fiel. Wenn man sah, wie sich die ganze Lebenshaltung des Kleinkindes gerade in den letzten Kriegsjahren so außerordentlich verschlechterte, wie zu den erschwerten Ernährungsverhältnissen, zu der mangelhaften Körperpflege und Unsauberkeit auch eine wesentliche Beschränkung an Licht, an Sonne und frischer Luft hinzutrat, bei den sich verschlechternden Wohnungsverhältnissen, der sich steigernden Kohlennot, so muß man sich eigentlich wundern, daß die Anzahl schwerer Fälle von englischer Krankheit nur um etwa $5 \%$; nicht um noch mehr zunahmen.

Es bleibt von den Konstitutionsanomalien des Kindes noch die exsudative Diathese zu besprechen. In den ersten Kriegsjahren, insbesondere 1916, konnte es scheinen, als ob ihre Manifestationen, 
insbesondere bei Säuglingen und Kleinkindern, etwas seltener geworden seien als zu Friedenszeiten. Wenigstens überwogen unter den Hautaffektionen dieser Altersstufe die Schmutzkrankheiten und Schmierinfektionen, wie die impetiginösen Ekzeme, über die rein exsudativen Zustände, den Milchschorf, Gneis, die Intertrigo und Prurigo, während sonst das Zahlenverhältnis dieser Affektionen eher umgekehrt war. Man konnte denken, daß die quantitative und qualitative Änderung der Kost der Kinder, die erzwungene Einschränkung in der Verabreichung von Eiern und Milch, überhaupt die Erschwerung der Überfütterung, die Manifestationen der exsudativen Diathese hatten seltener werden lassen.

Aber weiterhin war, ungeachtet der sich noch wesentlich verschlimmernden Ernährungsverhältnisse, wobei auch die Milch für die Kleinkinder erheblich verkürzt wurde, wieder deutlich eine $Z$ unah me der Erscheinungen dieser Konstitutionsanomalie festzustellen, in allen Altersklassen aller Bevölkerungsschichten, sowohl bei den Säuglingen und Kleinkindern meines poliklinischen Materials wie bei den Kindern aus der Volksschule wie auch bei den bemittelten Knaben in den Vorschulklassen und Unterstufen der Gymnasien und Realschulen. Eine Auszählung der Kleinkinder aus den unteren Bevölkerungsschichten mit floridem Gneis, Intertrigo, Strophulus u. a., mit Landkartenzunge oder hypertrophischen Tonsillen, andererseits eine Auszählung der älteren Knaben aus den oberen Ständen, aus den Gymnasien, mit den Residuen solcher Erscheinungen, mit tastbaren Hyperplasien der Lymphdrüsen der Halsregion, am Nacken oder unter dem Unterkiefer, ergab im Jahre 1918 in den meisten Gruppen größere Zahlen, oft um mehrere Prozent größere Zahlen, als die Zählung der entsprechenden gleichaltrigen Gruppen aus dem Jahre 1913. Bemerkenswert war die Häufigkeit, in der Schwellungen der Halslymphdrüsen noch bei den herangewachsenen Gymnasiasten, in der Mitte des zweiten Lebensdezenniums, angetroffen wurden; sonst waren es in dieser Altersperiode der von Haus aus gut gepflegten Knaben nur mehr Ausnahmen, jetzt aber eine häufige Erscheinung, wie sonst nur bei den jüngeren, hygienisch weniger gut gehaltenen Volksschülern. Die exsudativen Symptome waren nicht nur häufiger sondern auch hartnäckiger geworden.

An sich hat wohl diese Vermehrung einiger Konstitutionsanomalien, die größere Krankheitsbereitschaft zu Exsudationen oder zu neuropathischen Zuständen im Laufe der Kriegsjahre nicht allzuviel auf sich, 
mehr schon die, wenn auch an sich nur geringfügige Verschlechterung im allgemeinen konstitutionellen Verhalten, im gesamten Entwieklungszustand. Aber kommt hierzu nun auch noch ein selbst nur mäßiges Zurückbleiben im Körpergewicht, teils durch Hemmung des Wachstums, teils durch Verkleinerung des Fettdepots, durch den Aufbrauch der Fettreserven, und schließlich noch der Rückstand im Längenwachstum, was ich als die bemerkenswerteste Erscheinung betrachten möchte, so summieren sich damit in bedeutsamer Weise eine Reihe für die Gesundheit des Kindes nachteiliger, ungünstiger Umstände. Aus ihrem Zusammentreffen mit einer großen Zahl äußerer Schädlichkeiten, äußerer ungünstiger Momente resultiert die geringere Leistungsfähigkeit der Kinder in den letzten Kriegsjahren, die Herabsetzung ihrer Immunität, ihre geringere Widerstandskraft gegenüber Infektionen, überhaupt bei allen möglichen Erkrankungen.

Bei keinem Leiden zeigte sich dies stärker und schärfer als an dem Gang der Tuberkulose im Kindesalter während der Kriegsjahre. Zwei Perioden der Häufung, der Versehlimmerung, eines progredienten, oft kaum aufzuhaltenden Fortschreitens der Tuberkulose, sei es der Drüsen- oder der Knochengelenktuberkulose oder der LungenDarmtuberkulose, konnte ich an meinem Beobachtungs- und Krankenmaterial feststellen, zwei Perioden, die wohl zeitlich nachfolgend immerhin den beschriebenen Hemmungen und Minderungen einigermaßen parallel verliefen. Das war zum erstenmal vom Ausgang des Jahres 1916 bis zur Mitte 1917, wo ich eine ungewöhnlich große Reihe von Kindern und Jugendlichen zum Ende kommen sah, meist alte Patienten, die schon seit Jahren manifest tuberkulös erkrankt waren, die aber immer wieder die Exacerbation, das Aufflammen alter Herde gut oder doch leidlich überstanden hatten, aus eigener Kraft und begünstigt durch mannigfache Heilfaktoren, während sie jetzt erschöpft und von außen her nicht mehr ausreichend unterstützt der Infektion erlagen. Ein ähnlicher zweiter Schub erfolgte im Anschluß an die Grippe, am Fnde des Jahres 1918 und im Jahre 1919, nur daß hier die Tuberkulose im allgemeinen einen noch rascheren, oft gleich von vornherein progredienten Verlauf nahm.

Ein nach allen Richtungen abschließendes Urteil über die Bedeutung der geschilderten Wachstumshemmungen, der Einbußen und Minderungen, auf die Gesundheit des Kindes und der herangewachsenen Jugend dürfte sich erst nach einer Reihe von Jahren fällen lassen. 
112 E. Schlesinger: Wachstum, Gewicht und Konstitution der Kinder

1. Höhere Schulen.

\begin{tabular}{c|c|c|c|c|c|c|c|c}
\hline & \multicolumn{3}{|c|}{1911 und 1913} & \multicolumn{4}{|c}{19} \\
\cline { 2 - 9 } Alter & Anzahl & Länge & Gewicht & $\begin{array}{c}\text { Index } \\
\text { ponderal. }\end{array}$ & Anzahl & Länge & Gewicht & $\begin{array}{c}\text { Index } \\
\text { ponderal. }\end{array}$ \\
\hline 6 & 73 & 118,5 & 21 & 23,3 & 33 & 116,2 & 20,7 & 23,6 \\
7 & 246 & 122 & 21,5 & 22,8 & 122 & 121,5 & 22,5 & 23,2 \\
8 & 168 & 126,8 & 24,5 & 22,9 & 154 & 125,8 & 24,2 & 22,9 \\
9 & 260 & 132,6 & 26,9 & 22,6 & 195 & 131 & 26,9 & 22,8 \\
10 & 510 & 135,5 & 28,9 & 22,6 & 325 & 135 & 29 & 22,7 \\
11 & 442 & 139,9 & 31,4 & 22,5 & 355 & 139 & 30,8 & 22,5 \\
12 & 437 & 145,2 & 34,7 & 22,5 & 407 & 143,1 & 33,5 & 22,5 \\
13 & 537 & 150,5 & 38,8 & 22,5 & 363 & 149,8 & 37,9 & 22,4 \\
14 & 482 & 157,3 & 42,5 & 22,2 & 328 & 155,9 & 43,2 & 22,5 \\
15 & 358 & 163,4 & 48 & 22,2 & 334 & 161,8 & 48,4 & 22,5 \\
16 & 251 & 168,5 & 54,7 & 22,5 & 249 & 167,7 & 54,2 & 22,5 \\
17 & 177 & 170,5 & 59,4 & 22,9 & 168 & 169,9 & 56,7 & 22,7 \\
18 & 159 & 172 & 61,2 & 22,9 & 60 & 171,4 & 59,8 & 22,8
\end{tabular}

2. Gym nasium I (viele Knaben aus vermögenden Familien).

\begin{tabular}{r|r|lll|l|l|l|l|l}
6 & 30 & 120 & 21,8 & 23,3 & 15 & 118,5 & 21,4 & 23,4 \\
7 & 65 & 123 & 22,9 & 23 & 47 & 122,8 & 22,7 & 23 \\
8 & 38 & 128 & 25,4 & 22,9 & 46 & 127,6 & 24.1 & 22,6 \\
9 & 64 & 134 & 28,1 & 22,7 & 43 & 130,3 & 27 & 23 \\
10 & 91 & 137 & 29,4 & 22,5 & 68 & 137,1 & 29,9 & 22,6 \\
11 & 76 & 142 & 31,8 & 22,3 & 62 & 141,5 & 32,2 & 22,5 \\
12 & 76 & 148 & 35,5 & 22,2 & 53 & 144,1 & 33,6 & 22,4 \\
13 & 113 & 153 & 40,2 & 22,4 & 60 & 151,4 & 39,2 & 22,4 \\
14 & 71 & 158 & 42,7 & 22,1 & 57 & 158,4 & 45 & 22,4 \\
15 & 48 & 166 & 51,7 & 22,4 & 43 & 162,3 & 49 & 22,5 \\
16 & 45 & 172 & 57,5 & 22,4 & 55 & 169 & 55 & 22,5 \\
17 & 35 & 172 & 63,3 & 23,2 & 24 & 171,8 & 58,7 & 22,6 \\
18 & 27 & 174 & 62,3 & 22,8 & 8 & 171,4 & 62,3 & 23,1
\end{tabular}

3. Gymnasium III (katholisch, Mittelstand, sehr viele Knaben vom Lande).

\begin{tabular}{r|r|l|l|l|l|l|l|l}
7 & 12 & 125 & 23,4 & 22,9 & & & & \\
8 & 18 & 128 & 24,8 & 22,8 & & & & \\
9 & 36 & 133 & 27,8 & 22,8 & 22 & 131 & 28,9 & 23,4 \\
10 & 53 & 136 & 29 & 22,6 & 45 & 135 & 29,5 & 23 \\
11 & 47 & 140 & 30,9 & 22,4 & 45 & 138 & 30,6 & 22,7 \\
12 & 56 & 146 & 34,6 & 22,3 & 53 & 143 & 34,9 & 22,8 \\
13 & 94 & 152 & 38,5 & 22,2 & 60 & 149 & 37,7 & 22,5 \\
14 & 100 & 157 & 42,2 & 22,2 & 53 & 153 & 41,8 & 22,7 \\
15 & 81 & 161 & 49,6 & 22,8 & 82 & 159 & 48,1 & 22,9 \\
16 & 69 & 168 & 55,4 & 22,7 & 64 & 165 & 53,3 & 22,8 \\
17 & 62 & 169 & 59,6 & 23,1 & 46 & 167 & 55,8 & 22,9 \\
18 & 57 & 171 & 62,2 & 23,2 & 23 & 172 & 59,5 & 22,7
\end{tabular}


und der herangewachsenen Jugend während des Krieges.

4. Oberrealschule I (Vermögende und oberer Mittelstand).

\begin{tabular}{|c|c|c|c|c|c|c|c|c|}
\hline \multirow[b]{2}{*}{ Alter } & \multicolumn{4}{|c|}{1911 und 1913} & \multicolumn{4}{|c|}{1918} \\
\hline & Anzahl & Länge & Gewicht & $\begin{array}{c}\text { Index } \\
\text { ponderal. }\end{array}$ & Anzahl & Länge & Gewicht & $\begin{array}{c}\text { Index } \\
\text { ponderal. }\end{array}$ \\
\hline 6 & 13 & 118 & 21,1 & 23,4 & 9 & 114 & 20,5 & 24 \\
\hline 7 & 62 & 121 & 22 & 23,1 & 29 & 119 & 21,2 & 23,2 \\
\hline 8 & 37 & 127 & 24,6 & 22,9 & 46 & 126 & 24 & 23 \\
\hline 9 & 42 & 131 & 26,8 & 22,8 & 32 & 131 & 25,9 & 22,6 \\
\hline 10 & 93 & 135 & 28,5 & 22,6 & 52 & 136 & 28,9 & 22,6 \\
\hline 11 & 79 & 140 & 32,1 & 22,7 & 68 & 139 & 30,7 & 22,5 \\
\hline 12 & 73 & 145 & 34,2 & 22,4 & 79 & 145 & 33,3 & 22,2 \\
\hline 13 & 87 & 151 & 40,1 & 22,7 & 58 & 151 & 37,9 & 22,2 \\
\hline 14 & 83 & 159 & 43 & 22 & 51 & 158 & 44,8 & 22,5 \\
\hline 15 & 66 & 166 & 50 & 22,2 & 65 & 163 & 48,1 & 22,3 \\
\hline 16 & 38 & 170 & 54,3 & 22,3 & 38 & 169 & 54,9 & 22,5 \\
\hline 17 & 29 & 172 & 58 & 22,5 & 35 & 168 & 54,4 & 22,6 \\
\hline 18 & 34 & 173 & 61 & 22,8 & 9 & 169 & 57,9 & 22,9 \\
\hline
\end{tabular}

5. Oberrealschule II (Mittelstand und Schüler vom Lande).

\begin{tabular}{r|r|lll|l|l|l|l|l}
6 & 8 & 117 & 17,4 & 22,1 & & & & \\
7 & 42 & 119 & 21,6 & 23,4 & 22 & 121 & 22,8 & 23,4 \\
8 & 32 & 125 & 23,8 & 23 & 25 & 124 & 23,6 & 23,1 \\
9 & 54 & 131 & 27,2 & 23 & 43 & 127 & 25,8 & 23,2 \\
10 & 136 & 134 & 28,5 & 22,8 & 80 & 132 & 28,1 & 23 \\
11 & 111 & 139 & 31,5 & 22,6 & 83 & 137 & 29,8 & 22,6 \\
12 & 98 & 144 & 34,2 & 22,5 & 89 & 140 & 32,8 & 22,8 \\
13 & 105 & 149 & 37,5 & 22,4 & 87 & 146 & 38,1 & 23 \\
14 & 103 & 158 & 42,7 & 22,1 & 80 & 152 & 42,4 & 22,2 \\
15 & 83 & 161 & 47,4 & 22,4 & 63 & 155 & 48,2 & 23,5 \\
16 & 41 & 166 & 52,5 & 22,5 & 38 & 165 & 55,7 & 23,1 \\
17 & 24 & 169 & 56,9 & 22,8 & 28 & 168 & 56,4 & 23,8 \\
18 & 17 & 170 & 61,3 & 23,2 & 5 & 171 & 57,8 & 22,6
\end{tabular}

6. Realschule (Mittelstand).

\begin{tabular}{r|r|r|l|l|l|l|l|l|l}
9 & & & & & 7 & 130 & 27,6 & 23,2 \\
10 & 39 & 137 & 28,7 & 22,3 & 32 & 134 & 27,9 & 22,7 \\
11 & 65 & 140 & 31,5 & 22,5 & 52 & 139 & 30,6 & 22,6 \\
12 & 65 & 145 & 33,9 & 22,3 & 79 & 141 & 32,6 & 22,6 \\
13 & 61 & 149 & 37,3 & 22,3 & 61 & 147 & 36,5 & 22,5 \\
14 & 70 & 158 & 43,9 & 22,3 & 50 & 154 & 41,5 & 22,6 \\
15 & 45 & 164 & 48,6 & 22,2 & 53 & 162 & 48,4 & 22,5 \\
16 & 23 & 167 & 55,2 & 22,8 & 31 & 164 & 51,4 & 22,7 \\
17 & & & & & 8 & 170 & 57,1 & 22,6
\end{tabular}

Zeitschrift für Kinderheilkunde. 0 . XXII. 
114 E. Schlesinger: Wachstum, Gewicht und Konstitution der Kinder

7. Mittelsehule (gehobene Volksschule).

\begin{tabular}{r|c|c|c|c|c|c|c|c|c}
\hline \multirow{2}{*}{ Alter } & \multicolumn{4}{|c|}{1911} & und 1913 & \multicolumn{4}{|c|}{1918} \\
\cline { 2 - 10 } & Anzahl & Länge & Gewicht & $\begin{array}{c}\text { Index } \\
\text { ponderal. }\end{array}$ & Anzahl & Länge & Gewicht & $\begin{array}{c}\text { Index } \\
\text { ponderal. }\end{array}$ \\
\hline 9 & 159 & 130 & 26,6 & 22,9 & 38 & 128 & 24,3 & 22,6 \\
10 & 95 & 134 & 26,7 & 22,3 & 46 & 134 & 27,4 & 22,5 \\
11 & 55 & 140 & 31 & 22,4 & 47 & 137 & 29,3 & 22,5 \\
12 & 48 & 144 & 34,1 & 22,5 & 41 & 142 & 32,2 & 22,4 \\
13 & 51 & 151 & 36,9 & 22 & 57 & 146 & 34,6 & 22,3 \\
14 & 50 & 153 & 39,3 & 22,2 & 31 & 154 & 40,2 & 22,3
\end{tabular}

8. Volksschule (Unbemittelte).

\begin{tabular}{r|r|l|l|l|l|l|l|l}
6 & 85 & 113 & 19,2 & 23,7 & 42 & 111 & 18 & 23,6 \\
7 & 50 & 119 & 20,9 & 23,2 & 39 & 116 & 20,3 & 23,5 \\
8 & 37 & 123 & 22,6 & 23 & 38 & 124 & 22,9 & 22,9 \\
9 & 142 & 127 & 24,7 & 23 & 45 & 125 & 24,1 & 23 \\
10 & 83 & 131 & 27,1 & 22,9 & 27 & 130 & 26,2 & 22,8 \\
11 & 52 & 135 & 28,2 & 22,6 & 35 & 136 & 28,7 & 22,5 \\
12 & 48 & 141 & 32,3 & 22,5 & 26 & 141 & 31,1 & 22,3 \\
13 & 58 & 148 & 35,6 & 22,3 & 31 & 144 & 33,4 & 22,4
\end{tabular}

9. Hilfsschule für Schwachbegabte (meist Arme).

\begin{tabular}{r|r|rl|l|rll|l}
8 & 12 & 122 & 22,2 & 23 & 7 & 117 & 20,4 & 23,3 \\
9 & 10 & 121 & 23 & 23,5 & 22 & 119 & 22,2 & 23,6 \\
10 & 8 & 127 & 24,1 & 22,7 & 16 & 127 & 24,8 & 22,9 \\
11 & 6 & 134 & 25,9 & 22 & 8 & 131 & 26,5 & 22,7 \\
12 & 8 & 140 & 26,4 & 21,3 & 16 & 136 & 28 & 22,4 \\
13 & 13 & 136 & 34,9 & 24 & 17 & 143 & 32,5 & 22,2
\end{tabular}

10. Die gut entwickelten Knaben des Gymnasiums I.

\begin{tabular}{r|l|l|l|l|r|l|l|l}
6 & 11 & 124 & 24,4 & 23,4 & 6 & 120 & 22,6 & 23,5 \\
7 & 38 & 125 & 23,9 & 23 & 26 & 124 & 23,9 & 23 \\
8 & 27 & 131 & 26,6 & 22,9 & 25 & 128 & 24,5 & 22,6 \\
9 & 32 & 137 & 29,9 & 22,6 & 20 & 134 & 29,5 & 23,1 \\
10 & 51 & 140 & 31,9 & 22,7 & 35 & 140 & 32 & 22,7 \\
11 & 51 & 146 & 33,8 & 22,2 & 34 & 144 & 34,7 & 22,6 \\
12 & 48 & 150 & 37,9 & 22,3 & 28 & 146 & 35,4 & 22,5 \\
13 & 68 & 156 & 43,1 & 22,5 & 33 & 155 & 42,2 & 22,5 \\
14 & 38 & 160 & 46,4 & 22,4 & 33 & 163 & 49,5 & 22,6 \\
15 & 28 & 170 & 55 & 22,4 & 32 & 165 & 51 & 22,5 \\
16 & 27 & 171 & 59,5 & 22,8 & 36 & 171 & 58,7 & 22,7 \\
17 & 23 & 173 & 66,1 & 23,4 & 19 & 172 & 60,5 & 22,8 \\
18 & 11 & 175 & 68,2 & 23,4 & 6 & 173 & 64,6 & 23,2
\end{tabular}


und der herangewachsenen Jugend während des Krieges.

11. Die mittelmäßig entwickelten Knaben des Gymnasiums $I$.

\begin{tabular}{r|r|r|r|r|r|r|r|r}
\hline \multirow{2}{*}{ Alter } & \multicolumn{3}{|c|}{1911 und 1913} & \multicolumn{5}{|c|}{1918} \\
\cline { 3 - 8 } & Anzahl & Länge & Gewicht & $\begin{array}{c}\text { Index } \\
\text { ponderal. }\end{array}$ & Anzaht & Länge & Gewicht & $\begin{array}{c}\text { Index } \\
\text { ponderal. }\end{array}$ \\
\hline 6 & 7 & 120 & 19,6 & 22,5 & & & & \\
7 & 12 & 121 & 20,4 & 22,5 & 7 & 119 & 20,3 & 23 \\
8 & 6 & 122 & 21,5 & 22,7 & 5 & 125 & 22,7 & 22,6 \\
9 & 11 & 131 & 24,6 & 22,2 & 8 & 124 & 23,6 & 23,3 \\
10 & 15 & 133 & 25,8 & 22,3 & 10 & 132 & 27 & 22,7 \\
11 & 7 & 137 & 26,3 & 21,8 & 6 & 135 & 27,4 & 22,3 \\
12 & 13 & 142 & 29,9 & 21,8 & 12 & 139 & 29,8 & 22,3 \\
13 & 14 & 147 & 33 & 21,8 & 12 & 145 & 34,2 & 22,4 \\
14 & 12 & 147 & 35,9 & 22,4 & 5 & 150 & 37 & 22,2 \\
15 & 8 & 161 & 43,6 & 21,9 & 3 & 152 & 40 & 22,6 \\
16 & 6 & 172 & 51,2 & 21,6 & 4 & 151 & 39,4 & 22,5 \\
17 & 6 & 170 & 55,3 & 22,4 & & & & \\
18 & 5 & 172 & 55,1 & 22,2 & & & &
\end{tabular}

12. Die gut entwickelten Knaben der Realschule.

\begin{tabular}{r|rl|l|l|l|l|l|l}
9 & & & & & 8 & 132 & 28,2 & 23 \\
10 & 14 & 140 & 31,1 & 22,4 & 19 & 135 & 29,4 & 22,8 \\
11 & 38 & 143 & 33,5 & 22,6 & .18 & 141 & 32,7 & 22,7 \\
12 & 34 & 148 & 36,4 & 22,4 & 34 & 144 & 35,6 & 22,8 \\
13 & 26 & 153 & 41,6 & 22,6 & 26 & 151 & 39,2 & 22,4 \\
14 & 48 & 162 & 45,2 & 22,6 & 31 & 157 & 44,8 & 22,7 \\
15 & 28 & 167 & 52,2 & 22,4 & 35 & 165 & 51,6 & 22,6 \\
16 & 16 & 170 & 58,5 & 22,8 & 21 & 166 & 55 & 22,9 \\
17 & & & & & 10 & 173 & 61,2 & 22,8
\end{tabular}

13. Die mittelmäßig entwickelten Knaben der Realschule.

\begin{tabular}{l|r|r|l|l|r|l|l|l}
10 & 12 & 134 & 26,9 & 22,3 & 5 & 127 & 24,5 & 22,9 \\
11 & 6 & 135 & 27,5 & 22,4 & 8 & 132 & 26,3 & 22,6 \\
12 & 10 & 142 & 30,6 & 22 & 23 & 137 & 29 & 22,5 \\
13 & 8 & 141 & 31,8 & 22,4 & 12 & 139 & 31,4 & 22,7 \\
14 & 9 & 144 & 34,4 & 22,5 & 9 & 145 & 34,1 & 22,4 \\
15 & 7 & 162 & 43,8 & 21,8 & 8 & 150 & 39,4 & 22,7 \\
16 & 3 & 160 & 45,3 & 22,3 & 2 & 154 & 37,9 & 21,9
\end{tabular}


116 E. Schlesinger: Wachstum, Gewicht und Konstitution der Kinder

Das Verhaltender Konstitution. (Alle Zahlen in Prozenten.)

1. Höhere Schulen.

\begin{tabular}{|c|c|c|c|c|c|c|c|c|}
\hline \multirow{3}{*}{ Alter } & \multicolumn{4}{|c|}{4100 Knaben } & \multicolumn{4}{|c|}{3100 Knaben } \\
\hline & \multicolumn{4}{|c|}{1911 und 1913} & \multicolumn{4}{|c|}{1918} \\
\hline & gut & $\begin{array}{l}\text { gut bis } \\
\text { mittel- } \\
\text { mäßig }\end{array}$ & $\begin{array}{l}\text { mittel- } \\
\text { mäßig }\end{array}$ & $\begin{array}{c}\text { mangel- } \\
\text { haft }\end{array}$ & gut & $\begin{array}{l}\text { gut bis } \\
\text { mittel- } \\
\text { mäßig }\end{array}$ & $\begin{array}{l}\text { mittel- } \\
\text { mäBig }\end{array}$ & $\underset{\text { haft }}{\text { mangel- }}$ \\
\hline 6 & 38 & 29 & 30 & 3 & 43 & 39 & 15 & 3 \\
\hline 7 & 52 & 21 & 23 & 4 & 56 & 28 & 14 & 2 \\
\hline 8 & 57 & 24 & 17 & 2 & 59 & 23 & 17 & 1 \\
\hline 9 & 54 & 30 & 13 & 3 & 49 & 30 & 19 & 2 \\
\hline 10 & 55 & 26 & 17 & 2 & 59 & 25 & 14 & 2 \\
\hline 11 & 59 & 26 & 13 & 2 & 50 & 31 & 16 & 3 \\
\hline 12 & 58 & 24 & 16 & 2 & 51 & 25 & 23 & 1 \\
\hline 13 & 50 & 30 & 17 & 3 & 50 & 28 & 20 & 2 \\
\hline 14 & 51 & 26 & 20 & 3 & 56 & 26 & 15 & 3 \\
\hline 15 & 58 & 21 & 19 & 2 & 63 & 24 & 12 & 1 \\
\hline 16 & 53 & 30 & 17 & & 65 & 25 & 8 & 2 \\
\hline 17 & 55 & 25 & 19 & 1 & 70 & 26 & 3 & 1 \\
\hline 18 & 44 & 35 & 21 & & 64 & 26 & 10 & \\
\hline
\end{tabular}

2. Gymnasium I.

\begin{tabular}{r|r|rrr|r|r|r|r|r}
6 & 37 & 34 & 23 & 6 & 40 & 48 & 6 & 6 \\
7 & 59 & 20 & 18 & 3 & 55 & 26 & 15 & 4 \\
8 & 71 & 14 & 15 & & 54 & 33 & 11 & 2 \\
9 & 50 & 33 & 17 & & 48 & 30 & 18 & 4 \\
10 & 56 & 26 & 17 & 1 & 51 & 34 & 15 & \\
11 & 67 & 21 & 9 & 3 & 55 & 33 & 10 & 2 \\
12 & 64 & 18 & 17 & 1 & 53 & 25 & 22 & \\
13 & 60 & 26 & 12 & 2 & 56 & 25 & 19 & \\
14 & 53 & 27 & 18 & 2 & 57 & 32 & 9 & 2 \\
15 & 58 & 25 & 17 & & 73 & 19 & 8 & \\
16 & 59 & 27 & 14 & & 67 & 26 & 7 & \\
17 & 66 & 17 & 17 & & 78 & 22 & & \\
18 & 41 & 40 & 19 & & 75 & 13 & 12 &
\end{tabular}

3. Gymnasium III.

\begin{tabular}{r|r|r:r|r|r|r|r|r}
8 & 50 & 34 & 16 & & & & & \\
9 & 56 & 36 & 5 & 3 & 40 & 35 & 21 & 4 \\
10 & 57 & 22 & 21 & & 54 & 25 & 15 & 6 \\
11 & 46 & 29 & 21 & 4 & 33 & 36 & 31 & \\
12 & 57 & 18 & 23 & 2 & 52 & 24 & 24 & \\
13 & 54 & 26 & 17 & 3 & 41 & 35 & 22 & 2 \\
14 & 44 & 28 & 22 & 6 & 47 & 23 & 23 & 7 \\
15 & 63 & 17 & 19 & 1 & 62 & 21 & 16 & 1 \\
16 & 55 & 23 & 21 & 1 & 63 & 28 & 8 & 1 \\
17 & 55 & 26 & 16 & 3 & 71 & 27 & 2 & \\
18 & 40 & 39 & 21 & & 56 & 44 & &
\end{tabular}


und der herangewachsenen Jugend während des Krieges.

4. Oberrealschule $\mathrm{T}$.

\begin{tabular}{|c|c|c|c|c|c|c|c|c|}
\hline \multirow[b]{2}{*}{ Alter } & \multicolumn{4}{|c|}{1911 und 1913} & \multicolumn{4}{|c|}{1918} \\
\hline & gut & $\begin{array}{l}\text { gut bis } \\
\text { mittel- } \\
\text { mäBig }\end{array}$ & $\begin{array}{l}\text { mittel- } \\
\text { mäßig }\end{array}$ & $\begin{array}{c}\text { mangel- } \\
\text { haft }\end{array}$ & gut & $\begin{array}{l}\text { gut bis } \\
\text { mittel- } \\
\text { mäBig }\end{array}$ & $\begin{array}{l}\text { mittel- } \\
\text { mästg }\end{array}$ & $\begin{array}{c}\text { mangel- } \\
\text { haft }\end{array}$ \\
\hline 6 & 46 & 46 & 8 & & 67 & 11 & 22 & \\
\hline 7 & 46 & 17 & 34 & 3 & 56 & 24 & 17 & 3 \\
\hline 8 & 60 & 19 & 21 & & 56 & 17 & 24 & 3 \\
\hline 9 & 48 & 31 & 14 & 7 & 47 & 28 & 22 & 3 \\
\hline 10 & 57 & 25 & 16 & 2 & 59 & 17 & 8 & 6 \\
\hline 11 & 66 & 24 & 9 & 1 & 68 & 17 & 15 & \\
\hline 12 & 47 & 37 & 13 & 3 & 52 & 27 & 19 & 2 \\
\hline 13 & 46 & 30 & 23 & $I$ & 50 & 23 & 24 & 3 \\
\hline 14 & 45 & 30 & 25 & & 65 & 25 & 6 & 4 \\
\hline 15 & 52 & 24 & 23 & 1 & 57 & 32 & 8 & 3 \\
\hline 16 & 43 & 34 & 23 & & 68 & 24 & 5 & 3 \\
\hline 17 & 45 & 24. & $3 i$ & & 58 & 31 & 6 & 5 \\
\hline 18 & 35 & 35 & 30 & & 75 & 13 & 12 & \\
\hline
\end{tabular}

5. Oberrealschule II.

\begin{tabular}{r|rl|l|l|l|l|r|r}
7 & 52 & 30 & 16 & 2 & 64 & 27 & 9 & \\
8 & 56 & 25 & 16 & 3 & 56 & 16 & 28 & \\
9 & 55 & 28 & 17 & & 51 & 28 & 21 & \\
10 & 59 & 28 & 11 & 2 & 61 & 24 & 15 & \\
11 & 62 & 26 & 12 & & 55 & 21 & 19 & 5 \\
12 & 57 & 17 & 24 & 2 & 56 & 21 & 22 & 1 \\
13 & 42 & 38 & 16 & 4 & 56 & 23 & 19 & 2 \\
14 & 53 & 24 & 22 & 1 & 47 & 30 & 22 & 1 \\
15 & 53 & 18 & 21 & 8 & 58 & 26 & 14 & 2 \\
16 & 50 & 40 & 10 & & 68 & 19 & 11 & 2 \\
17 & 54 & 25 & 21 & & 55 & 30 & 15 & \\
18 & 53 & 35 & 12 & & & & &
\end{tabular}

6. Realschule.

\begin{tabular}{r|r|r|r|r|r|r|r|r}
10 & 36 & 28 & 31 & 5 & 59 & 26 & 15 & \\
11 & 58 & 30 & 9 & 3 & 34 & 48 & 16 & 2 \\
12 & 52 & 28 & 15 & 5 & 43 & 28 & 29 & \\
13 & 42 & 40 & 13 & 5 & 42 & 37 & 20 & 1 \\
14 & 69 & 17 & 13 & 1 & 62 & 16 & 18 & 4 \\
15 & 62 & 23 & 15 & & 67 & 18 & 15 & \\
16 & 69 & 18 & 13 & & 68 & 23 & 6 & 3
\end{tabular}


118 E. Schlesinger: Wachstum, Gewicht und Konstitution der Kinder

7. Mittelschule (gehobene Volksschule).

\begin{tabular}{r|c|c|c|c|c|c|c|c|c}
\hline & \multicolumn{3}{|c|}{1911 und 1913 } & \multicolumn{4}{|c|}{1918} \\
\cline { 2 - 12 } & gut & $\begin{array}{c}\text { gut bis } \\
\text { mittel- } \\
\text { mäBig }\end{array}$ & $\begin{array}{c}\text { mittel- } \\
\text { mäBg }\end{array}$ & $\begin{array}{c}\text { mangel- } \\
\text { haft }\end{array}$ & gut & $\begin{array}{c}\text { gut bis } \\
\text { mittel- } \\
\text { mäBig }\end{array}$ & $\begin{array}{c}\text { mittel- } \\
\text { mäBig }\end{array}$ & $\begin{array}{c}\text { mangel- } \\
\text { haft }\end{array}$ \\
\hline 9 & 63 & 21 & 16 & & 52 & 19 & 29 & \\
10 & 69 & 16 & 12 & 3 & 64 & 26 & 10 &. \\
11 & 62 & 22 & 16 & & 64 & 14 & 18 & 4 \\
12 & 50 & 28 & 20 & 2 & 70 & 19 & 11 & \\
13 & 58 & 22 & 20 & & 54 & 17 & 29 & \\
14 & 49 & 26 & 25 & & 59 & 22 & 17 & 2
\end{tabular}

8. Volksschule.

\begin{tabular}{r|r|r|r|r|r|r|r|r}
6 & 49 & 20 & 27 & 4 & 57 & 17 & 24 & 2 \\
7 & 61 & 24 & 15 & & 59 & 21 & 18 & 2 \\
8 & 40 & 34 & 24 & 2 & 64 & 23 & 13 & \\
9 & 53 & 18 & 27 & 2 & 51 & 21 & 26 & 2 \\
10 & 60 & 16 & 23 & 1 & 58 & 23 & 17 & 2 \\
11 & 42 & 18 & 38 & 2 & 60 & 21 & 15 & 4 \\
12 & 56 & 24 & 18 & 2 & 42 & 28 & 27 & 3 \\
13 & 58 & 21 & 16 & 5 & 41 & 22 & 34 & 3
\end{tabular}

9. Hilfsschule.

\begin{tabular}{r|r|rll|r|rrr|r}
8 & 43 & 33 & 16 & 8 & - & 75 & 25 & \\
9 & 20 & 60 & 10 & 10 & 24 & 31 & 40 & 5 \\
10 & 38 & 25 & 25 & 12 & 50 & 19 & 19 & 12 \\
11 & 34 & 33 & 33 & & 25 & 50 & 25 & \\
12 & 50 & 25 & 25 & & 12 & 44 & 44 & \\
13 & 61 & 16 & 13 & 10 & 29 & 41 & 24 & 6
\end{tabular}


und der herangewachsenen Jugend während des Krieges.

1. Auffallende Kleinheit der Statur.

(Alle Zahlen in Prozenten.)

\begin{tabular}{|c|c|c|c|c|c|c|c|c|c|c|}
\hline \multirow{2}{*}{ Alter } & \multicolumn{2}{|c|}{ Gymnasium I } & \multicolumn{2}{|c|}{ Gymnasium II } & \multicolumn{2}{|c|}{ Oberrealsehule } & \multicolumn{2}{|c|}{ Mittelschule } & \multicolumn{2}{|c|}{ Volksschule } \\
\hline & 1913 & 1918 & 1913 & 1918 & 1918 & 1918 & 1913 & 1918 & 1913 & 1918 \\
\hline 6 & 4 & - & 3 & 3 & 1 & 2 & & & 5 & 14 \\
\hline 7 & 2 & 3 & 2 & 6 & 4 & 6 & & & 4 & 1 \\
\hline 8 & 4 & 1 & 2 & - & 2 & 2 & & & 2 & - \\
\hline 9 & 3 & 8 & 1 & 2 & - & 2 & 4 & 10 & 5 & 3 \\
\hline 10 & 6 & 4 & 7 & 3 & 2 & 9 & 1 & 2 & 3 & - \\
\hline 11 & 6 & 3 & 5 & 5 & 3 & 3 & 4 & 3 & 4 & 3 \\
\hline 12 & 7 & 5 & 7 & 6 & 5 & 7 & 3 & 2 & 4 & 8 \\
\hline 13 & 8 & 3 & 5 & 10 & 5 & 9 & 1 & 5 & 4 & 8 \\
\hline 14 & 1 & - & 1 & 4 & 5 & 6 & 6 & 3 & & \\
\hline 15 & 2 & 3 & 1 & 2 & 3 & 3 & & & & \\
\hline 16 & - & 1 & & & & & & & & \\
\hline
\end{tabular}

2. Auffallende Schlankheit und GröBe.

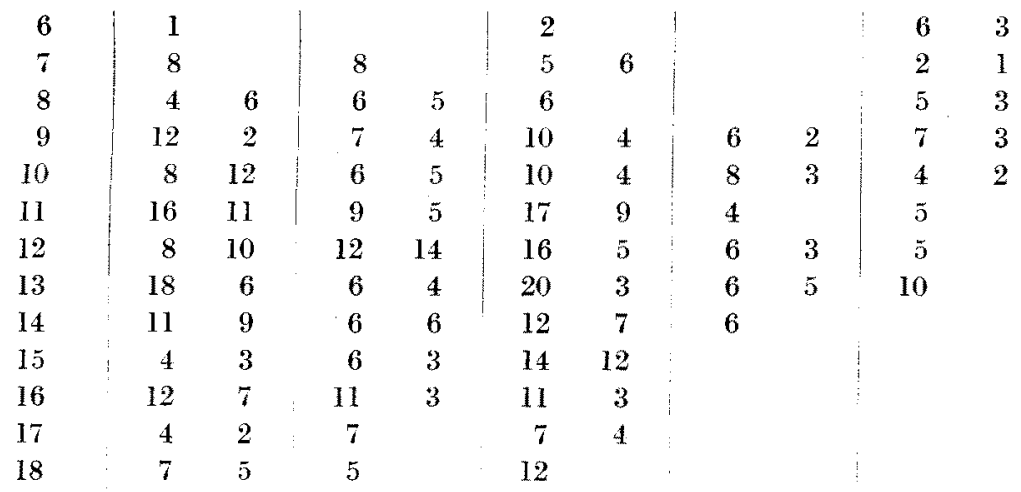

3. Auffallende Magerkeit.

\begin{tabular}{r|rr|rr|rr|rr|rr}
6 & 7 & 2 & 3 & 6 & & & & & 3 & 5 \\
7 & 10 & 3 & 7 & 2 & 10 & 12 & & & 3 & 3 \\
8 & 4 & 8 & 3 & 5 & 3 & 12 & & & 6 & 4 \\
9 & 20 & 15 & 3 & 8 & 8 & 6 & 4 & 6 & 5 & 6 \\
10 & 12 & 10 & 9 & 4 & 16 & 10 & 9 & 10 & 3 & 3 \\
11 & 13 & 12 & 7 & 8 & 5 & 8 & 3 & 6 & 7 & 5 \\
12 & 13 & 13 & 4 & 9 & 12 & 12 & 9 & 4 & 4 & 9 \\
13 & 10 & 7 & 6 & 4 & 15 & 9 & 3 & 9 & 10 & 3 \\
14 & 6 & 11 & 5 & 4 & 10 & 9 & 3 & 3 & & \\
15 & 5 & 3 & 4 & 4 & 8 & 11 & & & & \\
16 & 8 & 9 & 4 & 5 & 6 & 1 & & & & \\
17 & 2 & 3 & 6 & 4 & 7 & 4 & & & & \\
18 & & & 4 & 2 & 9 & 1 & & & &
\end{tabular}




\title{
Literatur über das Verhalten der Kinder während des Krieges.
}

\author{
Neugeborene.
}

Kettner-Charlottenburg, Kriegsneugeborene. Zeitschr. f. Säuglingsschutz 8, 329. 1916.

Langstein, Bendix, Misch, Dietrich-Berlin, Dasselbe. Ebenda 129, 335, $343,345$.

Tschirch - Jena, Zur Frage der Kriegsneugeborenen. Münch. med. Wochenschr. 1916, S. 1650.

De Waha - Erfurt, EinfluB des Krieges auf die Neugeborenen. Zeitschr. f. Säuglingsschutz 9, 386.1917.

Ham m - Straßburg, Kriegsneugeborene. Straßburger med. Ztg. 1917, Dez.

Peller - Wien, Maße der Neugeborenen und Kriegsernährung der Schwangeren. Deutsche med. Wochenschr. 1917, Nr. 6.

Brüning - Rostock, Zur Frage der Kriegsneugeborenen. Deutsche med. Wochenschr. 1918, S. 581.

Maron - München, Einfluß der Ernährungsverhältnisse im Kriege auf den körperlichen Entwicklungszustand der Neugeborenen. Veröff. d. Med.-Verw. 1918, 8. H., 7.

Binz-München, Krieg und Geburt. Münch. med. Wochenschr. 12. 1919.

\section{Säuglinge.}

Rott-Berlin, Einwirkung des Krieges auf die Säuglingssterblichkeit und Säuglingshewegung. Zeitschr. f. Säuglingssehutz 7, 177. 1915.

Benjamin - Cöln, Felsenthal - Mannheim, Guradze - Berlin, Dasselbe. Ebenda $1916, \$ .398,545,614$.

Noeggerath (- Brüssel), Einfluß desKrieges aufSäuglingsfürsorge und Säuglingssterblichkeit in Belgien. Zeitschr. f. Bevölkerungspolitik und Säuglingsfürsorge 1917 , S. 277.

He s s - Straßburg, Ernährungsbedingungen der Säuglinge im Krieg. Ebenda S. 47.

Steinhardt - Nürnberg, Vom Stillen in der Kriegszeit. Münch. med. Wochenschr. 1917, S. 943.

Opitz - Peine, Stillfähigkeit im Krieg. Deutsche med. Wochenschr. 1918, S. 437. Bachauer-Augsburg, Einwirkung des Krieges auf die Gesundheit der Jugend. Zeitschr. f. Schulgesundh. 1917, S 300 .

Schlesinger - Straßburg, Gewicht und Ernährung der Säuglinge und Kleinkinder im 3. Kriegsjahr. Zeitschr. f. Säuglingsschutz 10, 209. 1918.

\section{Kleinkinder.}

Noeggerath - Freiburg, Kleinkinder im Kriege in Baden. Jahrb. f. Kinderheilk. 1918, S. 272 und Zeitschr. f. Säuglingsschutz 1917, Oktober.

Gottstein-Charlottenburg, Gesundheitliche Versorgung der Kleinkinder im Krieg. Schriften des deutschen Ausschusses f. Kleinkinderfüirsorge 1917, Nr. 3. 
und der herangewachsenen Jugend wăhrend des Krieges.

Pfa undler- München. Kleinkinderkost im Krieg. Münch. med. Wochensehr. 1916, S. 1749.

Finkelstein - Berlin, Dasselbe. Ebenda 1917, \$. 112.

Die ganze Kindheit, insbesondere Schulkinder.

Schlossmann - Düssseldorf, Kinderkrankheiten und Krieg. 31. Vers. der Gesellschaft für Kinderheilkunde 1917. - Diskussion: Siegert - Cöln, BrüningRostock, Flachs-Dresden, Vogt - Magdeburg, Pei per - Greifswald, Thie misch - Leipzig, Rietschel - Würzburg, Noeggerath - Freiburg, Bernhard $\mathrm{t}$ - Berlin, Langstein - Charlottenburg.

Thiele-Chemnitz und Bachauer-Augsburg, Einwirkung des Krieges auf die Gesundheit der Jugend. Vers. des deutschen Vereins für Schulgesundheitspflege, Berlin 1917. Zeitschr. f. Schulgesundheitspflege 1917, S. 281. Diskussion: Roth - Braunschweig, Flachs - Dresden, Lewandowski - Berlin, Meyrich - Leipzig, Krautwig - Cöln, Seydel - Charlottenburg, Stein haus - Dortmund, Weber - Berlin, Beninde-Hertel - Berlin, Rabnow Schöneberg, Kehr - Düsselderf, Rotter - Darmstadt, A bel - Jena.

Roller - Gießen, Einwirkung des Krieges auf die Gesundheit der Jugend. Ebenda $1919, \$ 330$.

Czerny, Hamel, Beninde-Berlin, Abwehr einer bedrohlichen weiteren Verschlechterung unserer Ernährungsverhältnisse. Vereinigte ärztl. Gesellschaft, Berlin 1918, Dez. Berliner klin. Wochenschr. 1. 1919.

Hepner-Mannheim, Ernährungszustand der Schulanfänger 1915. Zeitschr. f. Sehulgesundh. 1915 , S. 545 .

Engelhorn - Göppingen, Zeitschr. f. Kinderforschung 1916.

Kettner-Charlottenburg, Das 1. Kriegsjahr und die großstädtischen Volksschulkinder. Deutsche med. Wochenschr. 1915, S. 1428.

Schlesinger-StraBburg, Einfluß der Kriegskost auf die schulpflichtige und herangewachsene Jugend. Münch, med. Wochenschr. 1917, S. 76 und Archiv f. Kinderheilk. 66, 161, 1917.

- Dasselbe in dritten Kriegsjahr. Miinch. med. Wochenschr. 1917, S. 1505.

Lebfeld - Magdeburg, Einfluß der Kriegsernährung auf die Magdeburger Volksschüler. Zeitschr. f. Schulgesundh. 1917, S. 33.

Haag - Heilbronn, Schulärztliche Erfahrungen in den Kriegsjahren 1916/17. Ebenda S. 225.

Kürbs - Eisenach. Dasselbe, Ebenda S. 46.

Sessler u. Honburger - Karlsruhe, Schulkinder während des Krieges und dic Kriegsernährung 1916/17. Fbenda, S. 441.

Griesbach - Straßburg, Einfluß der Kriegskost auf die Ernährung der Jugend. Deutsche med. Wochenschr. 1917, S. 722.

Oschmann - Weißenfels, Einfluß der Kriegskost auf die Schulkinder. Zeitschr. f. Schulgesundh. 1917, S. 32 .

Cle mens u. Thiele - Chemnitz, EinfluB der Kriegsernährung auf die Volksgesundheit. Münch. med. Wochenschr. 1917, S. 1082. 
Rosenfeld - Breslau, Kriegskost und Gesundheit. Berliner klin. Wochenschr 1918, Nr. 48 und Med. Klin. 1919, S. 225 und Berliner klin. Wochenschr. 1917, Nr. 28.

Birk - Kiel, Einfluß des Krieges auf die Kinder. Med. Klin. 1918, \$.127.

Häberlin - Wyk - Föhr, Körperliche Entwicklung des Kindes im Frieden und Krieg. Archiv f. Kinderheilk. 66, 370. 1918.

Ka u pe - Bonn, Einwirkungen des Krieges auf das Kind. Zeitschr. f. Nervenheilk. 60, 33. 1918.

Pfaundler - München, Ration und Bedarf an Nährstoffen für Kinder. Mïnch. med. Wochenschr. 1918, S. 173.

Herbst-Berlin, Stoffwechselversuche an kräftigen und schwächlichen Schulkindern bei Kriegskost. Jahrb. f. Kinderheilk. 1917, 85. H. 2.

Fuhge - Berlin, Stoffwechseluntersuchungen an Kindern im 3. Kriegsjahr. Ebenda 1918,88 . S. 43.

v. Met ten hei m - Frankfurt, Einfluß der Kriegskost auf die Häufung bestimmter Krankheitszustände im Kindesalter. Med. Klin. 1917, S. 645.

Weihe - Frankfurt, Dasselbe. Ebenda 1917, S. 476.

Rietschel - Würzburg, Kriegsenuresis und ihre Beziehungen zum Salz- und Kohlenhydratst offwechsel. Münch. med. Wochenschr. 1918, S. 693.

Kleinschmidt - Berlin, Prophylaxe der Kindertuberkulose im Krieg. Deutsche med. Wochenschr. 1917, S. 32.

Schäfer - Berlin, Zahnkrankheiten und Kriegsernährung. Berliner klin. Wochenschr. 1916, S. 1339.

Me yrich - Leipzig, Blutuntersuchungen an Jugendlichen. Pädagog.-physiolog. Arbeiten Leipzig 1918, 8 und Zeitschr. f. Schulgesundheitspflege 1917, S. 151.

Poetter - Leipzig, Dasselbe. Deutsche med. Wochenschr. 1917, Nr. 19.

Hildebrandt-Berlin, Kriegswirkungen im Seelenleben unserer Sohuler. Zeitschr. f. Schulgesundheitspflege 1918, S. 125.

Bernhard, Strelitz, Borbein, Benda-Berlin. Dasselbe. Ebenda 127.

\section{Die herangewachsene Jugend.}

Lommel - Jena, Einfluß der kriegsmäßig veränderten Ernährung. Deutsehe med. Wochenschr. 1916, S. 351.

Thiele - Chemnitz, Einfluß der kriegsmäßig veränderten. Ernährung auf unsere heranwachsende Jugend. Berliner klin. Wochenschr. 1916, S. 780.

Schlesinger, Bauchauer, Meyrich siehe oben.

\section{Andere angeführte Literatur.}

Aron, Wachstumsstörungen im Kindesalter. Jahrb. f. Kinderheilk. 87, 237. 1918.

Camerer, W. sen., Gewichts- und Längenwachstum des Menschen. Ebenda 36, 249, 1893 und 53, 381. 1901.

Martius, Konstitution und Vererbung in ihrer Beziehung zur Pathologie. Berlin 1914.

Mäller, Erich, Diagnostische und therapeutische Erwägungen bei blassen Zuständen im Kindesalter. Med. Klin. 1917, S. 353. 
und der herangew achsenen Jugend während des Krieges.

Pfaundler, Körpermaßstudien an Kindern. Zeitschr. f. Kinderheilk. 14, 1. 1916.

¥. Pirquet, Sitzhöhe und Körpergewicht. Zeitschr. f. Kinderheilk. I4, 211. 1916.

- Bestimmung des Ernährungszustandes. Ebenda 18, 220. 1918.

Schanz, Schule und Skoliose. Jahrb. f. Kinderheilk. 23, Erg. 1. 1911.

Schlesinger, Wachstum der Knaben und Jünglinge vom 6. bis 20. Jahr. Zeitschr. f. Kinderheilk. 16, 265.1917.

Stolte, Störungen des Längenwachstums der Säuglinge. Jahrb. f. Kinderheilk. 78, 399.1913.

Tobler u. Bessau, Allgemeine pathologische Physiologie der Ernährung und des Stoffwechsels im Kindesalter. 1914. 